\title{
Dynamics of spherically symmetric spacetimes: Hydrodynamics and Radiation
}

\author{
Marcelo Salgado * \\ Instituto de Ciencias Nucleares \\ Universidad Nacional Autónoma de México \\ Apdo. Postal 70-543 México 04510 D.F., México
}

\begin{abstract}
Using the $3+1$ formalism of general relativity we obtain the equations governing the dynamics of spherically symmetric spacetimes with arbitrary sources. We then specialize for the case of perfect fluids accompanied by a flow of interacting massless or massive particles (e.g. neutrinos) which are described in terms of relativistic transport theory. We focus in three types of coordinates: 1) isotropic gauge and maximal slicing, 2) radial gauge and polar slicing, and 3) isotropic gauge and polar slicing.
\end{abstract}

Submitted to Phys. Rev. D

*e-mail address: marcelo@nuclecu.unam.mx 


\section{INTRODUCTION}

One of the most fascinating phenomena in gravitational physics is that of gravitational collapse. Notably, the gravitational collapse of astrophysical bodies culminating in the formation of black holes.

The historical controversy on the final fate of gravitational collapse of compact objects such as white dwarfs and neutron stars raised by the discovery of maximum mass limits and the subsequent stability analysis led to find, at least for the most ideal configurations, definite answers and concrete predictions depending on the initial conditions and the equation of state of matter (see Refs. [1,2] for a review). The simplest situation describing the gravitational collapse ending in a black hole formation is that of a spherical ball of pressureless and homogeneous fluid (the well known Oppenheimer-Snyder dust collapse [3]). The solution shows the appearance of an event horizon revealing thus the formation of a black hole after a finite proper time.

Since that pioneering investigation, much has been done for more complicate and realistic initial configurations. On one hand, the accelerated development in the area of computation and the recent advances on the numerical analysis of Einstein equations have made possible computing the dynamics of rather complex spacetimes faster and for longer term evolutions. On the other hand, the advances in particle and nuclear physics have led to a better knowledge of the conditions of matter at high densities, providing then more realistic models for the matter in cores and neutron stars.

Most of the recent analysis of gravitational collapse leading to a black hole formation have been done in spherical symmetry and following a more "modern" point view in light of the $3+1$ formulation of general relativity and other formulations better adapted to numerical stability (see Ref. 44,5] and the references therein). Among these investigations, there is the one of Shapiro \& Teukolsky [6] who studied the collapse of polytropes by imposing an isotropic gauge and maximal slicing coordinates, with initial conditions provided by the Tolman-Oppenheimer- 
Volkoff equation of hydrostatic equilibrium. Later, a similar study, was performed by Schinder, Bludman and Piran [7] in comoving coordinates with a polar slicing, and by Gourgoulhon [8,9] in radial gauge and polar slicing, this latter improving previous analysis by the incorporation of realistic equations of state for the nuclear matter.

The effects of the coordinate choice and the slicing condition on the time evolution has been exhibited for two of the most popular choices (isotropic and radial gauges with maximal and polar slicings) by comparing with the analytical solution of Oppenheimer-Snyder (OS) 9 11]. For the time being, the discussion of gauges is postponed to Sec. VII.

The analysis of gravitational collapse of compact stars and iron cores would not be complete if the influence of neutrinos were not taken into account. This has been recognized by a long list of authors since the precursor investigations of Colgate and White [12], and May and White [13], who analyzed the effect of neutrinos in supernovae explosions. Later Wilson [14], performed full general relativity computations with a neutrino flow described in terms of the relativistic Boltzmann equation. Wilson's analysis included electron and muon massless neutrinos assuming that the corresponding antineutrinos contributed in the same basis. The interaction of neutrinos with the star's fluid was described by an opacity function. Unlike previous studies, Wilson's found that the heat conduction by neutrinos is not sufficient to eject any material from a collapsing star. In all those studies black hole formation were not analyzed but only evolution configurations terminating in stable states corresponding to white dwarfs or neutron stars.

An updated analysis were carried out by Mayle, Wilson and Schramm [15] using a Boltzmann code and for a large set of mass configurations. Neutrino signals from various species were analyzed within time scales of $\sim 1 \mathrm{~s}$ after the supernova explosion. It is to be mentioned that previously Saenz and Shapiro 16] had computed a non-spherical Quasi-Newtonian collapse acompanied by neutrino and gravitational radiation.

Burrows and coworkers have also analyzed during the last twenty years the mechanism 
of Type II supernovae (SNII) explosions and the role of neutrinos (see [17,18] and references therein). Among these investigations, we find an interesting model of long term neutrino emission from the hot protoneutron star phase to the final outcome of a stable cold neutron star $[19]$.

Many other recent investigations have confirmed and improved in several aspects previous findings on SNII (see [20 25] and references therein). For the case of a core collapse leading to a black hole two scenarios are recognized [22]. One called early black hole formation which is generically associated with accreting protoneutron stars which form from the collapse of degenerate cores of massive stars [25,26]. The accretion of some tenths to one solar mass can last a second, and the exceeding of the maximum mass drives the protoneutron star into a black hole collapse in a typical time scale of $\sim 0.5 \mathrm{~ms}$. In this scenario the neutrino signal is abruptly cut off after the black hole forms, and the typical neutrino luminosities prior the cutoff are $\sim 10^{52} \mathrm{erg} / \mathrm{s}$ per flavor.

The second scenario called late black hole formation typically arises by a softening of the high-density equation of state of the protoneutron star [27 30]. The phase transition from the neutron star matter to a more exotic state which include kaon condensates [31,32], or hyperon condensates [33] can lower the maximum allowable mass to $\sim 1.5 M_{\odot}$ [28, 30], driving thus a stable protoneutron star to an unstable regime and finally to a collapse into a black hole. This kind of core collapse can last $\sim 10 \mathrm{~s}$ before the cutoff and the luminosity of neutrinos is ten times lower than the luminosity of the early case.

It is encouraging to note that for a SNII at a distance of $\sim 10 \mathrm{kpc}$ which explodes within the early scenario, SuperKamiokande can probe $\bar{\nu}_{e}$ masses down to $1.8 \mathrm{eV}$ by comparing the arrival times of high and low energy neutrinos within the reaction $\bar{\nu}_{e}+p \rightarrow e^{+}+n$ in a Cerenkov detector (see Ref. [22] for details).

In fact the very recent announcement on the measurements of solar neutrinos from the decay of ${ }^{8} \mathrm{~B}$ by the Sudbury Neutrino Observatory (SNO) [34] via charged current interactions and by the elastic scattering of electrons reveals that neutrinos could be changing flavor as 
they travel from the sources to the Earth. This discovery if confirmed could corroborate the oscillating behavior of neutrinos and therefore their massive nature. The fluxes measured of the different flavors are in close agreement with the predictions of the solar models. The SNO experiment then implies that the upper limit of the mass squared difference between the $\nu_{e}$ and the $\nu_{\tau}$ or $\nu_{\mu}$ is less than $10^{-3} \mathrm{eV}^{2}$ [34]. This result when combined with the current bounds on $m_{\nu_{e}}$ of $2.8 \mathrm{eV}$ and $\Delta m_{\nu_{\mu} \nu_{\tau}}^{2}$ (assuming neutrino oscillations) provides a limit for the sum of the masses of the three neutrino species in the range $[0.05,8.4] \mathrm{eV}$ [34].

One proposal to measure the $\nu_{\tau}$ and $\nu_{\mu}$ masses indirectly and that can corroborate the SNO findings is the one which uses a time-of-flight technique [22], for neutrinos emitted in the early black hole formation scenario discussed above. The point is that if neutrinos are massive then there is a delay (relative to a massless neutrino) in the cutoff of the neutrino signal as measured on Earth after the black hole forms, and it is given by $\Delta t \sim\left(m_{\nu} / E_{\nu}\right)^{2}$ for distances of $\sim 10 \mathrm{kpc}$. This delay can affect the event rate measured in a detector. The conclusion is that assuming luminosities $L \sim 10^{52} \mathrm{erg} / \mathrm{s}$ per flavor at the cutoff time, SuperKamiokande can probe e-neutrino masses as small as $1.8 \mathrm{eV}$ for $T_{\nu_{e}} \sim 3.5 \mathrm{MeV}$, whereas the OMNIS [35] or SNO detector can detect $m_{\nu_{\mu}, \nu_{\tau}}$ masses as small as $6 \mathrm{eV}$ for $T_{\nu_{\mu}, \nu_{\tau}} \sim 8 \mathrm{MeV}$.

A collapse scenario which is rather different from the above consists in the accretion of matter by an old neutron star near the maximum mass limit. Gourgoulhon and Hansel [36] have analyzed the neutrino emission during the collapse to a black hole within this scenario via nonequilibrium $\beta$ processes, assuming that the nuclear matter is transparent for neutrinos (i.e., opacities were neglected). Instead of using neutrino transport, a regularized geometrical-optics model adapted to massless neutrinos was adopted. This model was thus intended to provide upper bounds in the neutrino burst. The collapse lasts typically a millisecond (the time that takes place the black hole formation), and in the most favorable conditions the total energy of $\bar{\nu}_{e}$ and $\bar{\nu}_{\mu}$ antineutrinos is $\sim 10^{51} \mathrm{erg}$, while the energy of the corresponding neutrinos is several orders of magnitude lower. This is even lower for the $\nu_{\tau}$ and $\bar{\nu}_{\tau}$ neutrinos. The main 
conclusion is that a collapse of this kind at a distance of $\sim 10 \mathrm{kpc}$ would be undetectable by the current neutrino detectors.

Finally, another scenario which has been analyzed in the past is the dynamics of collisionless gas of particles which mimic spherical star clusters, and the possibility of a cluster collapse into a supermassive black holes 38 42]. The motivation was to provide a theoretical description for the formation of supermassive black holes that could exist in the centers of galaxies. Recently, a similar study which include gamma-ray bursts, is the one analyzed by Linke et al. [43], where the collapse of supermassive stars $\left(M \sim 10^{5} M_{\odot}-10^{9} M_{\odot}\right)$ with emission of thermal neutrinos is considered. In that work, the spacetime is foliated by outgoing null hypersurfaces rather than using a $3+1$ foliation of spacetime.

In view of the different scenarios of gravitational collapse available today and the miscellaneous predictions within each of them it is worth pursuing the investigations along these lines. Only in this way there will be at hand a large set of models which the forthcoming (e.g. [35]) and recent observations [34] will validate or rule out.

Although the paper is written in the same spirit of various papers which deal with the system of equations Einstein-Hydrodynamics-Boltzmann, several aspects distinguish from them. For instance, most formalisms treat neutrinos as massless particles, except perhaps the one of Harleston and collaborators [44,45]. Here massive particles are considered from the onset and previous equations are recovered in the massless limit. The relativistic Boltzmann equation is written in terms of $3+1$ variables for generic spacetimes. This had done in the past only in spherical symmetry. Therefore, the relativistic Boltzmann equation presented here is coupled from the onset to the $3+1$ Einstein's equations. That is, the curvature effects appear in terms of the lapse, the shift, the extrinsic curvature and the three-metric. The hydrodynamic equations are derived also in the context of the $3+1$ formalism and they couple to the neutrinos via collision integrals. In particular the equation for the velocity field of the fluid is written in several forms each of one is useful whether one uses different numerical methods. At this 
regard, a general relativistic Euler equation is presented using the tetrad formalism. Its quasiNewtonian form allows an easy interpretation of several terms, and reduces to well known equations in various limits. Such an Euler equation turns to be better adapted for spectral methods than the equation for the momentum current density [8,9]. The system of equations are then specialized for spherical symmetry and written in three different coordinates: 1) isotropic gauge and maximal slicing, 2) radial gauge and polar slicing, and 3) isotropic gauge and polar slicing.

This is the first of a series of papers where the gravitational collapse of various kinds of matter will be analyzed.

The paper is organized as follows: in Sec. II we present succinctly the $3+1$ formalism of general relativity rather more to fix the notation than to give a detailed description. In Sec. III we consider the case of two interacting sources of matter: a perfect fluid and a flow of relativistic particles described in terms of relativistic transport theory. Section IV treats the relativistic transport theory. Section V deals with therodynamics. In section VI spherical symmetry is considered and in section VII three coordinate choices and slicing conditions are analyzed and discussed in light of the previous studies. Finally we conclude with some remarks and the plans for the forthcoming investigations along this line.

\section{THE 3+1 FORMALISM OF GENERAL RELATIVITY}

One of the most popular reformulations of general relativity when tackling numerical problems is the $3+1$ or Adison-Deser-Misner (ADM) formulation. We shall not enter into the details of the derivation of the equations (see Refs. [46 50]) but rather discuss the general idea in order to fix the notations.

The main idea is as follows: under general assumptions (see [47,50] and reference therein

for details) a globally hyperbolic spacetime $\left(M^{4}, g_{\mu \nu}\right)$ can be foliated by a family of spacelike hypersurfaces $\Sigma_{t}$ (Cauchy surfaces). Each hypersurface represents a Riemannian sub- 
manifold $\left(M^{3}, h_{i j}\right)$ endowed by an induced metric $h_{i j}$ (the 3 -metric). It is then assumed a local coordinate system $\left(t, x^{i}\right)$ for the spacetime, the spatial part $\left(x^{i}\right)$ represents a local coordinate system for $\Sigma_{t}$, while $t$ is a global time function that parametrizes $\Sigma_{t}$. The embedding of $\Sigma_{t}$ in spacetime is completed by the extrinsic curvature of $\Sigma_{t}$. This is defined by

$$
K_{\mu \nu}:=-\frac{1}{2} \mathcal{L}_{\boldsymbol{n}} h_{\mu \nu}
$$

where $\mathcal{L}_{\boldsymbol{n}}$ stands for the Lie derivative along the normal $n^{\mu}$ to $\Sigma_{t}$ and $h_{\mu \nu}:=g_{\mu \nu}+n_{\mu} n_{\nu}$. The vector field $n^{\mu}$ is time-like $\left(n^{\mu} n_{\mu}=-1\right)$ and the convention used for its components with respect to the coordinate base adapted to the spacetime foliation is as follows:

$$
n^{\mu}=\left(N, N^{i}\right)
$$

This convention means that $n^{\mu}$ points towards the future. Since $n^{\mu}$ is a unit time-like vector, it is customary to interpret $n^{\mu}$ as the four-velocity of the so-called Eulerian observer $\mathcal{O}_{\mathrm{E}}$. The scalar quantity $N$ (the lapse function) represents thus the rate at which $\mathcal{O}_{\mathrm{E}}$ sees the flow of its proper-time as compared with the intervals between two neighboring hypersurfaces $\Sigma_{t}$ and $\Sigma_{t+d t}$. The 3-vector $N^{i}$ (the shift-vector), represents the coordinate 3-velocity at which the Eulerian observer moves with respect to the coordinates $\left(t, x^{i}\right)$. In this way, the four-metric reads

$$
d s^{2}=-\left(N^{2}-N^{i} N_{i}\right) d t^{2}-2 N_{i} d t d x^{i}+h_{i j} d x^{i} d x^{j} .
$$

We emphasize that in many studies the convention on the sign of the shift vector is different from the one adopted here. This has to be taken into account particularly for purposes of comparison between the final form of equations in spherical symmetry presented here and the corresponding equations of the references which employs the opposite sign.

A useful formula for $K_{i j}$ obtained from Eq. (1) is

$$
K_{i j}=-\nabla_{i} n_{j}=-N \Gamma_{i j}^{t}=-\frac{1}{2 N}\left(\frac{\partial h_{i j}}{\partial t}+{ }^{3} \nabla_{j} N_{i}+{ }^{3} \nabla_{i} N_{j}\right)
$$

where ${ }^{3} \nabla_{j}$ stands for the covariant derivative compatible with $h_{i j}$. This is to be regarded as an evolution equation for the three-metric $h_{i j}$. 
The trace of the extrinsic curvature is simply given by,

$$
K:=-\nabla_{\alpha} n^{\alpha}
$$

Another useful quantity is the acceleration of $\mathcal{O}_{\mathrm{E}}$ given by

$$
a^{\mu}:=n^{\nu} \nabla_{\nu} n^{\mu}={ }^{3} \nabla^{\mu}[\ln N],
$$

which allows for the lapse the interpretation of the acceleration potential for $\mathcal{O}_{\mathrm{E}} 49$.

The orthogonal decomposition of the energy-momentum tensor in components tangent and orthogonal to $\Sigma_{t}$ leads to 49 :

$$
T^{\mu \nu}=S^{\mu \nu}+J^{\mu} n^{\nu}+n^{\mu} J^{\nu}+E n^{\mu} n^{\nu}
$$

The tensor $S^{\mu \nu}$ is symmetric and often called the tensor of constraints; $J^{\mu}$ is the momentum density vector and $E$ is the total energy density measured by the Eulerian observer $\mathcal{O}_{\mathrm{E}}$. Both $S^{\mu \nu}$ and $J^{\mu}$ are orthogonal to $n^{\mu}$.

We emphasize that for the specific applications we will study, $T^{\mu \nu}$ will be the total energymomentum tensor of matter which can be composed by the contribution of different types of sources:

$$
T^{\mu \nu}=\sum_{i} T_{i}^{\mu \nu}
$$

This means that

$$
E=\sum_{i} E_{i}, J^{\mu}=\sum_{i} J_{i}^{\mu}, S^{\mu \nu}=\sum_{i} S_{i}^{\mu \nu}
$$

The projection of Einstein equations $R_{\mu \nu}=4 \pi G_{0}\left(2 T_{\mu \nu}-T_{\alpha}^{\alpha} g_{\mu \nu}\right)$ in the directions tangent and orthogonal to $\Sigma_{t}$, followed by the use of the Gauss-Codazzi-Mainardi equations leads to the $3+1$ form of Einstein equations:

$$
{ }^{3} R+K^{2}-K_{i j} K^{i j}=16 \pi G_{0} E
$$

known as the Hamiltonian constraint. 


$$
{ }^{3} \nabla_{l} K^{l}{ }_{i}-{ }^{3} \nabla_{i} K=8 \pi G_{0} J_{i}
$$

known as the momentum constraint equations.

Finally, the dynamic Einstein equations read

$$
\begin{aligned}
& \partial_{t} K^{i}{ }_{j}+N^{l} \partial_{l} K^{i}{ }_{j}+K^{i}{ }_{l} \partial_{j} N^{l}-K^{l}{ }_{j} \partial_{l} N^{i}+{ }^{3} \nabla^{i 3} \nabla_{j} N-{ }^{3} R_{j}^{i} N-N K K_{j}^{i} \\
& =4 \pi G_{0} N\left[(S-E) \delta_{j}^{i}-2 S_{j}^{i}\right]
\end{aligned}
$$

where $S=S_{l}^{l}$ is the trace of the tensor of constraints, and all the quantities written with a ' 3 ' index refer to those computed with the three-metric $h_{i j}$. Moreover, under the $3+1$ formalism tensor quantities tangent to $\Sigma_{t}$ use the three-metric to raise and lower their spatial indices. The equations (41) and (12) are the set of the Cauchy-initial-data evolution equations for the gravitational field subject to the constraints Eqs. (10) and (11).

An evolution equation for the trace $K$ is obtained by taking the trace in Eq. (12):

$$
\partial_{t} K+N^{l} \partial_{l} K+{ }^{3} \Delta N-N\left({ }^{3} R+K^{2}\right)=4 \pi G_{0} N[S-3 E]
$$

where ${ }^{3} \Delta$ stands for the Laplacian operator compatible with $h_{i j}$.

This can be simplified by using Eq.(10) to give

$$
\partial_{t} K+N^{l} \partial_{l} K+{ }^{3} \Delta N-N K_{i j} K^{i j}=4 \pi G_{0} N[S+E]
$$

To complete the system of equations, we consider also the matter equations

$$
\nabla_{\mu} T^{\mu \nu}=0
$$

which according with Eq. (8), these can be written as

$$
\nabla_{\mu} T_{\psi}^{\mu \nu}=-\mathcal{F}^{\nu}
$$

where $T_{\psi}^{\mu \nu}$ is the energy-momentum tensor of certain fields that are collectively labeled by $\psi$ and $\mathcal{F}_{\nu}:=\nabla_{\mu} T_{\text {ext }}^{\mu \nu}$ are the "forces" exerted by the external fields (fields other than $\psi$ ). For instance, we shall consider the case where the total energy-momentum tensor is given by a combination of a perfect-fluid and a radiated flow of particles: 


$$
T^{\mu \nu}=T_{\mathrm{PF}}^{\mu \nu}+T_{\mathrm{R}}^{\mu \nu}
$$

so that $T_{\psi}^{\mu \nu}=T_{\mathrm{PF}}^{\mu \nu}$ and $T_{\mathrm{ext}}^{\mu \nu}=T_{\mathrm{R}}^{\mu \nu}$; thus, the energy-momentum tensor of the perfect-fluid alone will not conserve by separate; $\mathcal{F}_{\nu}$ will represent the "forces" of the radiated flow acting on the perfect fluid in form of collisions.

The energy-momentum conservation equations (16) can be written in $3+1$ form as well. The projection of Eq. (16) along $n^{\mu}$ leads to the energy conservation equation,

$$
\partial_{t} E_{\psi}+N^{l} \partial_{l} E_{\psi}+\frac{1}{N}{ }^{3} \nabla_{l}\left(N^{2} J_{\psi}^{l}\right)=N\left(S_{\psi}^{i j} K_{i j}+E_{\psi} K\right)+N n_{\nu} \mathcal{F}^{\nu}
$$

Explicitly $n_{\nu} \mathcal{F}^{\nu}=-N \mathcal{F}^{t}$

On the other hand, the projection of Eq. (16) on $\Sigma_{t}$ leads to the momentum conservation equation,

$$
\partial_{t} J_{i}^{\psi}+N^{l} \partial_{l} J_{i}^{\psi}+J_{l}^{\psi} \partial_{i} N^{l}+N\left({ }^{3} \nabla_{l \psi} S^{l}{ }_{i}\right)=N K J_{i}^{\psi}-\left({ }_{\psi} S^{l}{ }_{i}+E_{\psi} \delta^{l}{ }_{i}\right)^{3} \nabla_{l} N-{ }^{3} \mathcal{F}_{i} N
$$

where

$$
{ }^{3} \mathcal{F}_{i}=h_{i \mu} \mathcal{F}^{\mu}=-N_{i} \mathcal{F}^{t}+h_{i j} \mathcal{F}^{j}
$$

\section{A. Tetrads}

In many applications the use of tetrad components of tensors (hereafter physical components) are better adapted to a problem than the coordinate components. This will be the case when writing the equations of relativistic transport for the radiated flow and the equations of motion for the matter.

In the context of the $3+1$ formalism the tetrad we use is the local tetrad of the Eulerian

observer which is given by $\left\{n^{\mu}, e_{(i)}^{j}\right\}$, that is, by the time-like vector normal to $\Sigma_{t}$ and by a triad on $\Sigma_{t}$. In covariant notation that tetrad is given by:

$$
e_{(\mu)}=q_{(\mu)}^{\nu} \frac{\partial}{\partial x^{\nu}}
$$


where $\partial / \partial x^{\nu}$ denotes the coordinate basis of the spacetime, and $q_{(\mu)}^{\nu}$ are the tetrad coefficients that allow the normalization. For instance, it turns that $e_{(t)}^{\mu} \equiv n^{\mu}$.

The inverse relationship of Eq. (21) is given by

$$
\frac{\partial}{\partial x^{\mu}}=e_{\mu}^{(\nu)} e_{(\nu)}
$$

where the coefficients $e_{\mu}^{(\nu)}$ are related to $q_{(\mu)}^{\nu}$ by the completeness relations $e_{\nu}^{(\alpha)} q_{(\beta)}^{\nu}=\delta_{(\beta)}^{(\alpha)}$, and $e_{\nu}^{(\alpha)} q_{(\alpha)}^{\mu}=\delta^{\mu}{ }_{\nu}$.

A tetrad is not uniquely defined. The Lorentz invariance $\mathrm{SO}(3,1)$ leaves the freedom on the choice of the six parameters that rotate and boost frames. As mentioned, a convenient choice is as follows,

$$
\begin{aligned}
& e_{t}^{(i)}:=-e_{j}^{(i)} N^{j}, \\
& e_{i}^{(t)}:=0, \\
& e_{t}^{(t)}=N, \\
& h_{i j}=e_{i}^{(l)} e_{j}^{(l)} .
\end{aligned}
$$

The inverse relations are,

$$
\begin{aligned}
& q_{(t)}^{i}=\frac{N^{i}}{N}, \\
& q_{(i)}^{t}=0, \\
& q_{(t)}^{t}=N^{-1}, \\
& h^{i j}=q_{(l)}^{i} q_{(l)}^{j} .
\end{aligned}
$$

here $e_{i}^{(l)} q_{(l)}^{j}=\delta^{i}{ }_{j} e_{l}^{(i)} q_{(j)}^{l}=\delta_{(j)}^{(i)}$.

This choice of a tetrad is compatible with the $3+1$ decomposition of the four-metric (3), so that

$$
d s^{2}=e_{\mu}^{(\alpha)} e_{\nu}^{(\beta)} \eta_{(\alpha)(\beta)} d x^{\mu} d x^{\nu}
$$

with $e_{\nu}^{(\alpha)}$ given by Eqs. (23)-(26) and $\eta_{(\alpha)(\beta)}$ stands for the Minkowski metric. 
Trivial Lorentz transformations of the chosen tetrad relates the Eulerian observer with other possible frames. Moreover, the best choice for a triad $e_{i}^{(l)}$ on $\Sigma_{t}$ will depend on the particular coordinates used on the hypersurface $\Sigma_{t}$ (see Sec. VI for the case of spherical symmetry).

Finally, the transformation law for the components of tensors tangent to $\Sigma_{t}$ from the coordinate base to the triad is as follows,

$$
\begin{aligned}
N^{(i)} & =e_{l}^{(i)} N^{l}, \\
J_{(i)} & =q_{(i)}^{l} J_{l}, \\
K_{(j)}^{(i)} & =e_{l}^{(i)} q_{(i)}^{m} K_{m}^{l}, \\
S_{(j)}^{(i)} & =e_{l}^{(i)} q_{(i)}^{m} S_{m}^{l} .
\end{aligned}
$$

The inverse relationships are obtained from above in the obvious way. The triad indices [i.e., spatial indices within '( )'] are raised and lowered with $\delta_{(j)}^{(i)}($ i.e., the triad-covariant and triadcontravariant components of 3-tensors are identical to each other). Four-tensor components transform in a similar way using the four-dimensional tetrad coefficients and $\eta_{(\mu)(\nu)}$ (resp. $\left.\eta^{(\mu)(\nu)}\right)$ to lower (resp. raise) indices.

The use of the tetrad formalism will be useful to recast the $3+1$ matter equations and later on to write the relativistic Boltzmann equation in a useful manner. For instance Eq. (18) reads,

$$
\partial_{(t)} E_{\psi}+{ }^{3} \nabla_{(i)} J_{\psi}^{(i)}-E_{\psi} K+2 J_{\psi}^{(i)}{ }^{3} \nabla_{(i)} \nu-{ }_{\psi} S^{(i)(j)} K_{(i)(j)}=-\mathcal{F}^{(t)}
$$

where $\partial_{(t)}=n^{\mu} \partial_{\mu}$.

On the other hand, the momentum conservation equation (19) can be written as

$\partial_{(t)} J_{\psi}^{(i)}+{ }^{3} \nabla_{(j)} S_{\psi}^{(i)(j)}+\left[S_{\psi}^{(i)(j)}+E_{\psi} \delta^{(i)(j)}\right]{ }^{3} \nabla_{(j)} \nu-J_{\psi}^{(i)} K+J_{\psi}^{(l)}\left(\mathcal{O}_{(t)(l)}^{(i)}-K_{(l)}^{(i)}\right)=-{ }^{3} \mathcal{F}^{(i)}$

We remind that the covariant-derivative components with respect to a tetrad uses the four-Ricci rotation coefficients (RRC) as a connection. We define them as follows, 


$$
\mathcal{O}_{(\beta)(\gamma)}^{(\alpha)}:=e_{\mu}^{(\alpha)} q_{(\beta)}^{\nu} \nabla_{\nu} q_{(\gamma)}^{\mu}=q_{(\beta)}^{\sigma} e_{\mu}^{(\alpha)}\left(\partial_{\sigma} q_{(\gamma)}^{\mu}+q_{(\gamma)}^{\lambda} \Gamma_{\lambda \sigma}^{\mu}\right)
$$

The three-RRC have an identical expression by restricting the above definition to pure spatial indices and using the three-covariant derivative. While the above definition requires the Christoffel symbols, these can be avoided by using the representation of the RRC in terms of the structure constants 48,51,52].

Although the $3+1$ Einstein equations can be written following a tetrad approach [48], for our purposes this will not be necessary and thus we will not pursue the issue here.

\section{PERFECT FLUIDS WITH SOURCES}

For the specific applications we have in mind, a combination of a perfect-fluid and a radiated flow will be considered. Then in Eq. (17) we assume

$$
T_{\mathrm{PF}}^{\mu \nu}=(\rho+p) u^{\mu} u^{\nu}+p g^{\mu \nu}
$$

For the moment the form of the radiating part $T_{\mathrm{R}}^{\mu \nu}$ is not specified. This will be treated in detail in Sec. IV.

The corresponding $3+1$ matter variables of the fluid are,

$$
\begin{aligned}
E_{\mathrm{PF}} & =(\rho+p) \Gamma-p, \\
J_{\mathrm{PF}}^{(i)} & =\left(E_{\mathrm{PF}}+p\right){ }^{3} U^{(i)}, \\
S_{\mathrm{PF}}^{(i)(j)} & =\left(E_{\mathrm{PF}}+p\right){ }^{3} U^{(i)}{ }^{3} U^{(i)}+\delta^{(i)(j)} p, \\
S_{\mathrm{PF}} & =\left(E_{\mathrm{PF}}+p\right)\left({ }^{3} U^{(i)}\right)^{2}+3 p, \\
\Gamma & :=-n_{\mu} u^{\mu}=u^{(t)}=\left[1-\left({ }^{3} U^{(i)}\right)^{2}\right]^{-1 / 2},
\end{aligned}
$$

where

$$
\begin{aligned}
{ }^{3} U^{(i)} & :=\frac{u^{(i)}}{\Gamma}=\frac{e_{l}^{(i)}}{N}\left(V^{l}-N^{l}\right)=\frac{1}{N}\left(V^{(l)}-N^{(l)}\right), \\
V^{l} & :=u^{l} / u^{t} .
\end{aligned}
$$


The equation for conservation of energy (36) applied to a perfect fluid with sources then reads $48,52,54$

$$
\partial_{(t)} E+{ }^{3} \nabla_{(l)}\left[(E+p){ }^{3} U^{(l)}\right]+(E+p)\left[2{ }^{3} U^{(j)} a_{(j)}-{ }^{3} U^{(l)}{ }^{3} U^{(j)} K_{(l)(j)}-K\right]=-\mathcal{F}^{(t)} .
$$

On the other hand, the momentum conservation equation (37) applied to a perfect-fluid with sources can be written as an Euler equation for the velocity field. This reads 44, 52, 54,

$$
\begin{gathered}
\partial_{(t)}{ }^{3} U^{(i)}+{ }^{3} U^{(j)}{ }^{3} \nabla_{(j)}{ }^{3} U^{(i)}=-\frac{1}{E_{\mathrm{PF}}+p}\left[{ }^{3} \partial^{(i)} p+{ }^{3} U^{(i)} \partial_{(t)} p\right]-a_{(j)} \\
+{ }^{3} U^{(i)}{ }^{3} U^{(l)}\left(a_{(l)}-{ }^{3} U^{(j)} K_{(l)(j)}\right)-{ }^{3} U^{(l)}\left(\mathcal{O}_{(t)(l)}^{(i)}-K_{(l)}^{(i)}\right)+\frac{1}{E_{\mathrm{PF}}+p}\left({ }^{3} U^{(i)} \mathcal{F}^{(t)}-\mathcal{F}^{(i)}\right) .
\end{gathered}
$$

where

$$
a_{(i)}={ }^{3} \nabla_{(i)}[\ln N]
$$

are the physical components of Eq.(6). This Euler equation is the version in physical components (with the extra dissipative terms $\mathcal{F}^{(\mu)}$ ) of Eq. (2.29) of Ref. [54] (see also Ref. [55]).

\section{RELATIVISTIC TRANSPORT THEORY}

We shall consider the phenomenon of relativistic transport of massive and massless particles (hereafter radiation) within a dense medium. We shall follow closely the formalism developed by Lindquist [56], but we will not repeat the details here. The radiated particles of the specie ' $R$ ' will be treated classically as point particles except when interacting with the dense medium. The interactions and its quantum mechanical effects will be ultimately translated as emission rates and opacity functions. The particles will be characterized thus by a four-momentum

$$
p^{\mu}=\frac{d x^{\mu}}{d \lambda}
$$

where $d \lambda$ corresponds to an affine parameter (for massless particles) or to the proper-time per mass unit (for massive particles). 
According to this formalism, one postulates the existence of a scalar function $F_{\mathrm{R}}\left(x^{\mu}, p^{\mu}\right)$ (the invariant distribution function for the specie $\mathrm{R}$ ) which is a function from the phase space coordinates $\left(x^{\mu}, p^{\mu}\right)$ to the reals. Actually, since we will be interested in particles satisfying the mass shell condition

$$
g_{\mu \nu} p^{\mu} p^{\nu}=-\tilde{m}
$$

where $\tilde{m}=0,1$ for massless or massive particles respectively, $F_{\mathrm{R}}$ will be a function defined on the reduced phase-space.

The invariant distribution function so introduced will be such that the number density four-vector and the energy-momentum tensor of the particles are respectively [56],

$$
\begin{aligned}
j_{\mathrm{R}}^{\mu} & =\int p^{\mu} F_{\mathrm{R}}\left(x^{\mu}, p^{\mu}\right) d P . \\
T_{\mathrm{R}}^{\mu \nu} & =\int p^{\mu} p^{\nu} F_{\mathrm{R}}\left(x^{\mu}, p^{\mu}\right) d P .
\end{aligned}
$$

where $d P$ is the invariant volume of the momenta space on shell [56:

$$
d P=-N \sqrt{h} \frac{d^{3} p}{p_{t}}
$$

where $d^{3} p:=d p^{1} d p^{2} d p^{3}$ represents a coordinate 3 -volume element and $h$ is the determinant of the 3-metric.

The distribution function $F_{\mathrm{R}}$ is related to the dimensionless distribution function $\bar{F}_{\mathrm{R}}$ by

$$
F_{\mathrm{R}}=\frac{g_{\mathrm{R}}}{8 \pi^{3} \hbar_{\mathrm{Pl}}^{3}} \bar{F}_{\mathrm{R}}
$$

where $g_{\mathrm{R}}$ is the statistical weight of the particles of the specie $\mathrm{R}$ (e.g., $g=1,2$ for neutrinos and photons respectively).

As in the case of the perfect fluid, it is useful to refer the components of four-momenta to a tetrad. In the context of the $3+1$ formalism we have,

$$
\begin{gathered}
e:=p^{(t)}=N p^{t}, \\
p^{(i)}=e_{l}^{(i)}\left(p^{l}-p^{t} N^{l}\right),
\end{gathered}
$$


with the inverse relationships given by

$$
\begin{aligned}
& p^{t}=e / N, \\
& p^{i}=e \frac{N^{i}}{N}+q_{(l)}^{i} p^{(l)},
\end{aligned}
$$

where $p^{(i)}$ are the physical spatial components of the 4-momentum (i.e. the spatial physical components of the projection of $p^{\mu}$ onto $\left.\Sigma_{t}:{ }^{3} p^{\mu}:=h^{\mu}{ }_{\nu} p^{\nu}\right)$. The ratio $p^{(i)} / e$ corresponds to the local velocity of particles measured by $\mathcal{O}_{\mathrm{E}}$.

Introducing the magnitude of the three-momentum as,

$$
p^{2}:=p_{(i)} p^{(i)}
$$

it is easy to see that (51) simply becomes

$$
e^{2}=p^{2}+\tilde{m}^{2}
$$

Here $e$ is the energy (per mass-unit in the case of massive particles) as measured by the Eulerian observer. Therefore, from Eq. (54), one obtains,

$$
d P=\sqrt{h} \frac{d^{3} p}{e\left(1+\frac{p_{(l)} e_{i}^{(l)} N^{i}}{E N}\right)} .
$$

where we used that $p_{t}=e_{t}^{(\mu)} p_{(\mu)}=N p_{(t)}-p_{(l)} e_{i}^{(l)} N^{i}=-N e-p_{(l)} e_{i}^{(l)} N^{i}$.

When changing variables in the momentum space using (59) and (61) a straightforward manipulations show that

$$
d^{3} p=\frac{d p^{(1)} d p^{(2)} d p^{(3)}}{\sqrt{h}}\left(1+\frac{p_{(l)} e_{i}^{(l)} N^{i}}{e N}\right)
$$

where it was used the fact that $\operatorname{det}\left[q_{(l)}^{i}\right]=1 / \sqrt{h}$. Then finally

$$
d P=\frac{d p^{(1)} d p^{(2)} d p^{(3)}}{e}
$$

which has exactly the same form of flat spacetimes.

The use of physical spherical variables in momentum space leads to 


$$
d P=p^{2} d p d \Omega_{p} / e=p d e d \Omega_{p}
$$

Indeed, this is the useful expression when dealing with spherical symmetry.

The use of tetrad components allows us to write Eqs. (52) and (53), as follows,

$$
\begin{aligned}
j_{\mathrm{R}}^{(\mu)} & =\int p^{(\mu)} F_{\mathrm{R}}\left(x^{\lambda}, p^{(\lambda)}\right) d P, \\
T_{\mathrm{R}}^{(\mu)(\nu)} & =\int p^{(\mu)} p^{(\nu)} F_{\mathrm{R}}\left(x^{\lambda}, p^{(\lambda)}\right) d P .
\end{aligned}
$$

Therefore, the corresponding $3+1$ matter variables are

$$
\begin{aligned}
E_{\mathrm{R}} & =\int e^{2} F_{\mathrm{R}}\left(x^{\lambda}, p^{(\lambda)}\right) d P, \\
J_{\mathrm{R}}^{(i)} & =\int e p^{(i)} F_{\mathrm{R}}\left(x^{\lambda}, p^{(\lambda)}\right) d P, \\
S_{\mathrm{R}}^{(i)(j)} & =\int p^{(i)} p^{(j)} F_{\mathrm{R}}\left(x^{\lambda}, p^{(\lambda)}\right) d P, \\
S_{\mathrm{R}} & =\int p^{2} F_{\mathrm{R}}\left(x^{\lambda}, p^{(\lambda)}\right) d P .
\end{aligned}
$$

According to (9) the total $3+1$ matter variables are,

$$
\begin{gathered}
E=E_{\mathrm{PF}}+E_{\mathrm{R}}, \\
J^{(i)}=J_{\mathrm{PF}}^{(i)}+J_{\mathrm{R}}^{(i)}, \\
S^{(i)(j)}=S_{\mathrm{PF}}^{(i)(j)}+S_{\mathrm{R}}^{(i)(j)}, \\
S=S_{\mathrm{PF}}+S_{\mathrm{R}} .
\end{gathered}
$$

where we remind that the quantities labeled with ' $\mathrm{PF}$ ' are given by Eqs. (40)-(43). It is understood in these expressions that the sum of the different quantities extend to all the species R considered.

\section{A. The Boltzmann equation in curved space-times}

We defined a "macroscopic" 4-current density number of particles of the specie R by Eq. (52). The number density of particles as measured in the local frame of an observer $\mathcal{O}_{p}$ with four-velocity $\left.v^{\mu}\right)$ is 


$$
n_{p}^{\mathrm{R}}:=-v_{\mu} j_{\mathrm{R}}^{\mu}=-\int v_{\mu} p^{\mu} F_{\mathrm{R}}\left(x^{\lambda}, p^{(\lambda)}\right) d P
$$

Therefore, the number of particles $d N_{\mathrm{R}}$ with momenta between $p^{\mu}$ and $p^{\mu}+d p^{\mu}$ crossing the volume element $d V$ of the space-like hypersurfaces orthogonal to $v^{\mu}$ and which is centered at some point $x^{\mu}$ of spacetime is

$$
d N_{\mathrm{R}}=F_{\mathrm{R}}\left(x^{\lambda}, p^{(\lambda)}\right)\left(-v_{\mu} p^{\mu}\right) d V d P
$$

The quantity $d W=\left(-v_{\mu} p^{\mu}\right) d V d \lambda$ represents the four-volume spanned by the flow of particles (world lines) crossing $d V$, which is given by the element of hypersurface $d V$ with normal $v^{\mu}$ and the particle's infinitesimal displacement orthogonal to $d V$ given by $d l=v_{\mu} p^{\mu} d \lambda$ [56]. The quantity $\left(-v_{\mu} p^{\mu}\right) d V$ is in fact the correct Lorentz invariant four-volume element. From the relativistic form of Liouville's Theorem (see Ref. [56] for the details) $d W d P$ remains invariant along a given set of trajectories. Therefore, the change in the number of world lines within $d W d P$ is proportional to the change in $F_{\mathrm{R}}$

$$
\begin{aligned}
\delta\left(d N_{\mathrm{R}}\right) & =\left[\frac{\partial F_{\mathrm{R}}}{\partial x^{\alpha}} d x^{\alpha}+\frac{\partial F_{\mathrm{R}}}{\partial p^{\alpha}} d p^{\alpha}\right]\left(-v_{\mu} p^{\mu}\right) d V d P \\
& =\left[\frac{\partial F_{\mathrm{R}}}{\partial x^{\alpha}} p^{\alpha}+\frac{d p^{\alpha}}{d \lambda} \frac{\partial F_{\mathrm{R}}}{\partial p^{\alpha}}\right] d W d P
\end{aligned}
$$

The evolution for $p^{\mu}$ will be thus governed by the equations of motion of individual particles:

$$
\frac{d p^{\mu}}{d \lambda}=-p^{\sigma} p^{\nu} \Gamma_{\sigma \nu}^{\mu}+\mathcal{F}_{\text {fields }}^{\mu}+\mathcal{F}_{\text {coll }}^{\mu}
$$

This equations shows that the acceleration of the particles is due to 1) the space-time curvature 2) the forces arising from the interaction of the particles with fundamental fields other than the gravitational one, 3) the interaction with other particles that can be represented by "collisions". For our purposes, we will consider that $\mathcal{F}_{\text {fields }}^{\mu}=0$. That is to say, the only fundamental field we consider is the gravitational one. All other interactions like the weak ones (in the case of neutrinos) will be treated phenomenologically as collision terms, and therefore, the set of equations will not include gauge-fields, but rather involve macroscopic quantities 
that characterize the medium and which are obtained from field theory in a similar fashion as one obtains the equation of state of the matter.

The relativistic Boltzmann equation (RBE) then reads,

$$
\hat{\boldsymbol{L}} F_{\mathrm{R}}=\left(\frac{d F_{\mathrm{R}}}{d \lambda}\right)_{\text {coll }}
$$

where

$$
\hat{\boldsymbol{L}}:=p^{\mu} \frac{\partial}{\partial x^{\mu}}-p^{\nu} p^{\sigma} \Gamma_{\nu \sigma}^{\mu} \frac{\partial}{\partial p^{\mu}}
$$

is the relativistic Liouville operator often written by the fuzzy notation $p^{\alpha} D / d x^{\alpha}$ (the direc-

tional derivative of $F_{\mathrm{R}}$ along the phase flow), and $\left(\frac{d F_{\mathrm{R}}}{d \lambda}\right)_{\text {coll }}=-\mathcal{F}_{\mathrm{R} \text { coll }}^{\alpha} \frac{\partial F_{\mathrm{R}}}{\partial p^{\alpha}}$ represents collectively the scattering, absorption and emission processes between the particles of the specie $\mathrm{R}$ and the medium. In the absence of collisions, the distribution function remain constant along the particle's path (i.e., along particle's geodesics). In the language of differential geometry, the operator $D / d x^{\alpha}=\frac{\partial}{\partial x^{\alpha}}-p^{\lambda} \Gamma_{\alpha \lambda}^{\mu} \frac{\partial}{\partial p^{\mu}}$ corresponds to a coordinate basis vector of the horizontal part of the tangent space of the bundle $\mathcal{B}$ over the spacetime $M^{4}$ [56]. It is important to emphasize that the mass shell condition implies that the distribution function will not be defined over the entire tangent space but only on that part where $p_{\mu} p^{\mu}=-\tilde{m}^{2}$, that is the distribution function will be defined only on the sphere bundle (the subbundle of tangent vectors of fixed length). One can incorporate this restriction in the Liouville operator by treating the spatial part $p^{i}$ of the four-momenta as independent components. Then

$$
\left(D / d x^{\alpha}\right)_{\mathrm{ms}}=\frac{\partial F_{\mathrm{R}}}{\partial x^{\alpha}}-p^{\lambda} \Gamma_{\alpha \lambda}^{i} \frac{\partial F_{\mathrm{R}}}{\partial p^{i}}
$$

\section{B. Tetrad representation and the $3+1 \mathrm{RBE}$}

It will be convenient to use a tetrad components to re-write the RBE. By employing the tetrad formalism it's easy to show that the RBE reads 


$$
\left(p^{(\alpha)} q_{(\alpha)}^{\mu} \frac{\partial}{\partial x^{\mu}}-p^{(\beta)} p^{(\alpha)} \mathcal{O}_{(\beta)(\alpha)}^{(\delta)} \frac{\partial}{\partial p^{(\delta)}}\right) F_{\mathrm{R}}\left(x^{\alpha}, p^{(\sigma)}\right)=\left(\frac{d F}{d \lambda}\right)_{\text {coll }}
$$

Let us consider Eq.(33), and split it in terms of temporal and spatial contributions. First we define,

$$
L_{p}:=p^{(b)} p^{(a)} \mathcal{O}_{(b)(a)}^{(d)} \frac{\partial}{\partial p^{(d)}}=p^{(b)} p^{(a)} \mathcal{O}_{(b)(a)}^{(t)} \frac{\partial}{\partial e}+p^{(b)} p^{(a)} \mathcal{O}_{(b)(a)}^{(i)} \frac{\partial}{\partial p^{(i)}}
$$

where the notation $e=p^{(t)}$ was used. The properties of the RRC and some straightforward calculations show a useful relationship between the four-RRC and the physical components of $3+1$ variables. For instance:

$$
\begin{aligned}
& \mathcal{O}_{(t)(i)}^{(t)}=\mathcal{O}_{(t)(t)}^{(i)}=a_{(i)}, \\
& \mathcal{O}_{(i)(j)}^{(t)}=\mathcal{O}_{(j)(t)}^{(i)}=-K_{(i)(j)}, \\
& \left.\mathcal{O}_{(t)(\mu)}^{(\mu)}=\mathcal{O}_{(i)(\mu)}^{(\mu)}=0 \text { (no sumation on } \mu\right) \\
& \mathcal{O}_{(t)(j)}^{(i)}=-\frac{1}{2}\left(-\frac{\partial_{(i)} N^{(j)}}{N}+q_{(m)}^{l} \frac{N^{(m)}}{N} \partial_{(i)} e_{l}^{(j)}+q_{(i)}^{l} \partial_{(t)} e_{l}^{(j)}-(i) \longleftrightarrow(j)\right) \\
& \mathcal{O}_{(i)(j)}^{(l)}={ }^{3} \mathcal{O}_{(i)(j)}^{(l)}
\end{aligned}
$$

Here ${ }^{3} \mathcal{O}_{(i)(j)}^{(l)}$ are the 3 -Ricci rotation coefficients, i.e., the RRC associated to the local basis frame on $\Sigma_{t}$, and $\partial_{(t)}=\frac{1}{N} \frac{\partial}{\partial t}+\frac{N^{(i)}}{N} \partial_{(i)}$.

In this way we obtain

$$
L_{p}=\left(e p^{(i)} a_{(i)}-p^{(i)} p^{(j)} K_{(i)(j)}\right) \frac{\partial}{\partial e}+\left(e^{2} a^{(i)}+e p^{(i)} \mathcal{O}_{(t)(j)}^{(i)}-e p^{(j)} K_{(j)}^{(i)}+p^{(l)} p^{(j) 3} \mathcal{O}_{(i)(j)}^{(l)}\right) \frac{\partial}{\partial p^{(i)}}
$$

Finally, the $3+1$ decomposition of Eq. (83) is,

$$
\begin{aligned}
& {\left[e \partial_{(t)}+p^{(i)} \partial_{(i)}-\left(e p^{(i)} a_{(i)}-p^{(i)} p^{(j)} K_{(i)(j)}\right) \frac{\partial}{\partial e}\right.} \\
& \left.-\left(e^{2} a^{(l)}+e p^{(i)} \mathcal{O}_{(t)(j)}^{(i)}-e p^{(j)} K_{(j)}^{(i)}+p^{(i)} p^{(j)}{ }^{3} \mathcal{O}_{(i)(j)}^{(l)}\right) \frac{\partial}{\partial p^{(l)}}\right] F_{\mathrm{R}}\left(x^{\alpha}, p^{(c)}\right)=\left(\frac{d F}{d \lambda}\right)_{\text {coll }} .
\end{aligned}
$$

It is to be noted that the properties of RRC imply that ${ }^{3} \mathcal{O}_{(i)(l)}^{(l)}$ (no sum convention) are null. Therefore, the above equation can be further simplified.

Equation (87) is the $3+1$ version of the of RBE, here written in terms of physical components. The mass shell condition $e^{2}=p^{(i)} p_{(i)}+\tilde{m}^{2}$ can be imposed on the RBE by considering, 
for instance, $p^{(i)}$ as independent variables. In that case $F_{\mathrm{R}}$ is to be considered as though it does not depend explicitly on $e$, i.e., $\partial F_{\mathrm{R}} / \partial e=0$. Alternatively, the use of spherical-like variables in momentum space (see next section) will allow us to consider $e$ as independent variable and $p^{2}=p^{(i)} p_{(i)}$ as the dependent one.

\section{Collisions}

As we stressed before, particles may be submitted to collision forces arising from the interacting medium (in the case of neutrinos these forces come from weak interactions with baryons). Let us remind that the collision integral as conceived originally by Boltzmann assumes that the interacting medium has a known distribution function. That's it, the distribution function of the medium is a data of the problem.

In the present case, the interacting medium is to be considered not as particles but rather as a fluid field, namely a perfect fluid. Thus, for our purpose, it will be more convenient to characterize the collision integral in terms of scalar functions as it is usual in transport theory. In this way collisions will be represented macroscopically by the so-called invariant opacity $o\left(x^{\mu}, p^{\mu}\right)$ and the invariant emissivity $\Upsilon\left(x^{\mu}, p^{\mu}\right)$.

We then assume that the collision integral takes the following form

$$
\left(\frac{d F_{\mathrm{R}}}{d \lambda}\right)_{\text {coll }}=\Upsilon-o F_{\mathrm{R}}
$$

In terms of quantities measured in the same frame one can write

$$
\Upsilon=\frac{\eta\left(x^{\mu}, p^{\mu}\right)}{e^{2}}=\frac{\eta^{\prime}\left(x^{\prime \mu}, p^{\prime \mu}\right)}{e^{\prime 2}}
$$

here $\eta$ and $e$ being the matter emissivity and the particle energy respectively, both measured in the same frame. In the same way, one can introduce the matter opacity as

$$
o=e \chi\left(x^{\mu}, p^{\mu}\right)=e^{\prime} \chi^{\prime}\left(x^{\prime \mu}, p^{\prime \mu}\right)
$$


where $\chi \sim 1 / \ell, \ell$, being the mean free path of the particle in the corresponding frame.

The collision term takes then the useful form

$$
\left(\frac{d F_{\mathrm{R}}}{d \lambda}\right)_{\text {coll }}=e\left(\frac{\eta}{e^{3}}-\chi F\right)
$$

The opacity $\chi$ and the absorption coefficient $\kappa$ are related by

$$
\chi=\kappa n,
$$

where $n$ is the proper number density of particles that composes the medium (e.g., the baryon density), such that $n:=-j^{\mu} u_{\mu}$ where $j^{\mu}$ is the four-current of baryons.

In this way, an alternative form of the collision integral is

$$
\left(\frac{d F_{\mathrm{R}}}{d \lambda}\right)_{\text {coll }}=\kappa_{e} n\left(\mathcal{S}-F_{\mathrm{R}}\right)
$$

where $k_{e}=e \kappa$, and $\mathcal{S}=\Upsilon / o$ is usually referred to as the effective source function.

It is to be emphasized that quantities measured in different frames are related to each other via the invariant quantities and Lorentz transformations, for instance, the relationship between the opacities meaured in the Eulerian frame and those of the proper frame of the fluid are given, according to Eq. (90), by

$$
e \chi=e_{p} \chi_{p}
$$

where quantities in the left-hand-side (l.h.s) refer to the Eulerian frame, while the quantities in the right-hand-side (r.h.s) refer to the proper frame of the fluid. Since physical components of four vectors in both frames are related by a Lorentz transformation, for instance

$$
p_{p}^{(\mu)}=\Lambda_{(\nu)}^{(\mu)^{p}} p^{(\nu)}
$$

where

$$
\left(\Lambda_{(\nu)}^{(\mu)^{p}}\right)=\left(\begin{array}{cc}
\Gamma & -{ }^{3} U^{(i)} \Gamma \\
-{ }^{3} U^{(i)} \Gamma & \delta_{(j)}^{(i)}+{ }^{3} U^{(i)} 3 U^{(j)} \frac{\Gamma^{2}}{\Gamma+1}
\end{array}\right)
$$

with ${ }^{3} U^{(i)}$ given by Eq.(45) and $\Gamma$ by Eq.(44), then for the time components, 


$$
e_{p}=\Gamma\left(e-{ }^{3} U^{(i)} p^{(i)}\right)=e \Gamma\left(1-{ }^{3} U^{(i)} v^{(i)}\right)=e \Gamma\left(1-\left\|{ }^{3} U^{(i)}\right\|\left\|v^{(i)}\right\| \cos \left(\theta_{\mathrm{R}}\right)\right),
$$

where $v^{(i)}:=p^{(i)} / e$ represents the velocity of the particles with respect to the Eulerian frame. In the last formula one recognizes the well known formula for the energy shift due to the relative motion of observers. The type of shift (red or blue) will depend on the angle $\theta_{\mathrm{R}}$ between the propagation vector of the particles $v^{(i)}$ and the velocity of the fluid ${ }^{3} U^{(i)}$ (i.e., blue or red shift if the fluid is approaching or receeding respectively from the Eulerian observer). Therefore the transformation formula between opacities yields

$$
\chi=\chi_{p} \Gamma\left(1-\left\|{ }^{3} U^{(i)}\right\|\left\|v^{(i)}\right\| \cos \left(\theta_{\mathrm{R}}\right)\right)
$$

In the case of massless particles $\left\|v^{(i)}\right\|=1$. In a similar way one can obtain the transformation formulae for the absortion coefficients and the emissivities.

\section{Conservation equations for the radiated flow}

The particle number current and the energy-momentum tensor of the radiated particles was introduced by Eqs. (52) and (53) respectively. For instance, in the case of perfect quantum gases in thermal equilibrium (i.e., Fermi and Bose gases) the above definitions allows one to recover the usual macroscopic expressions for the energy-density, density-number and pressure parameterized by the temperature, particle-mass and chemical potential of the species as measured in the local frame.

When collisions are present, both the particle number and the energy-momentum tensor of the particles will not conserve alone since there will be exchange of energy and momentum with the interacting dense fluid. Thus we can expect that the conservation equations derived from Eqs. (52) and (53) will have sources arising from the collision integral:

$$
\nabla_{\nu} j_{\mathrm{R}}^{\nu}=\int\left(\frac{d F}{d \lambda}\right)_{\text {coll }} d P
$$

and 


$$
\nabla_{\nu} T_{\mathrm{R}}^{\mu \nu}=\int p^{\mu}\left(\frac{d F}{d \lambda}\right)_{\text {coll }} d P
$$

Then we write

$$
\begin{aligned}
\nabla_{\nu} j_{\mathrm{R}}^{\nu} & =\mathcal{R}_{\mathrm{R}}, \\
\nabla_{\nu} T_{\mathrm{R}}^{\mu \nu} & =-\mathcal{F}_{\mathrm{R}}^{\mu}=n w_{\mathrm{R}}^{\mu},
\end{aligned}
$$

where

$$
\begin{aligned}
\mathcal{R}_{\mathrm{R}} & :=\int \kappa\left(x^{\mu}, p^{\mu}\right)\left[\mathcal{S}\left(x^{\mu}, p^{\mu}\right)-F_{\mathrm{R}}\left(x^{\mu}, p^{\mu}\right)\right] d P, \\
w_{\mathrm{R}}^{\mu} & :=\int p^{\mu} \kappa\left(x^{\mu}, p^{\mu}\right)\left[\mathcal{S}\left(x^{\mu}, p^{\mu}\right)-F_{\mathrm{R}}\left(x^{\mu}, p^{\mu}\right)\right] d P .
\end{aligned}
$$

One can define the mean emissivity (energy/(volumextime) in the fluid frame as

$$
\mathcal{D}:=-u_{\mu} \nabla_{\nu} T_{\mathrm{R}}^{\mu \nu}=-n u_{\mu} w^{\mu}
$$

Since we have been using quantities measured in the Eulerian frame, in the above expressions we are to use the corresponding quantities with respect to the same observer. Namely, the particle number density $n_{\mathrm{E}}$ as measured by the Eulerian observer is related to the proper number density of the perfect fluid $n$ by $n_{\mathrm{E}}=n \Gamma$. Same considerations apply for the remaining collision variables. For instance, the emissivity measured in the Eulerian frame $\mathcal{D}_{E}=-n_{\mu} n w^{\mu}=n w^{(t)}$

is related to the proper emissivity $\mathcal{D}=-u_{\mu} n w^{\mu}$ by $\mathcal{D}=\Gamma \mathcal{D}_{E}\left(1-{ }^{3} U^{(i)}{ }^{3} W^{(i)}\right)$ where ${ }^{3} W^{(i)}:=w^{(i)} / w^{(t)}$.

\section{THERMODYNAMICS}

In this section the thermodynamical description of the dense matter with which the radiative particles interact will be presented taking into account the mean quantities introduced in the previous section. Such a description is performed in the proper frame of the fluid. We then assume that the equation of state of the dense matter (i.e., the perfect fluid) is given in parametrized form as follows 


$$
\begin{aligned}
& \rho=\rho\left(s, n_{1}, \ldots, n_{m}\right), \\
& p=p\left(s, n_{1}, \ldots, n_{m}\right),
\end{aligned}
$$

where $s$ is the entropy density and $n_{M}(1 \leq M \leq m)$ is the number density of particles of the specie $M$ (e.g. baryons and the different lepton flavors), all of them measured in the fluid frame. For instance, in the case of a dense matter in hydrostatic equilibrium composed by a mixture of neutrons, protons and electrons, the electron density is obtained directly from the proper baryon density $n$ by demanding charge neutrality and chemical equilibrium $\mu_{\mathrm{n}}=\mu_{\mathrm{p}}+\mu_{\mathrm{e}}$. This last condition arising from the equilibirum of the nuclear reactions: $n \rightleftharpoons p+e^{-}$[9]. In that case the equation of state depends on $n$ solely.

Now, the equations (106) and (107) are not independent from each other but linked through the first principle of thermodynamics

$$
d \mathrm{U}=\theta d \mathrm{~S}-p d V+\mu^{\mathrm{R}} d \mathrm{~N}^{\mathrm{R}}
$$

where $\Theta$ and $\mu^{\mathrm{R}}$ are the temperature and the chemical potential of the specie $\mathrm{R}$ of the particles composing the fluid respectively, defined by

$$
\begin{aligned}
\Theta & =\left(\frac{\partial \rho}{\partial s}\right)_{n_{\mathrm{R}}}, \\
\mu^{\mathrm{R}} & =\left(\frac{\partial \rho}{\partial n_{\mathrm{R}}}\right)_{s, n_{B} \neq n_{\mathrm{R}}} .
\end{aligned}
$$

Using these definitions, Eq. (108) takes the following form in terms of densitized quantities [9],

$$
p=\Theta s+\mu^{\mathrm{R}} n_{\mathrm{R}}-\rho .
$$

This equation is often referred to as the compatibility themodynamic condition between Eqs. (106) and (107) [9].

The conservation equation for the baryon number reads,

$$
\nabla_{\mu} j^{\mu}=0
$$


where we remind that $j^{\mu}=n u_{\mu}$ is the density current of baryons and $n$ the proper baryon number density.

This equation can be written explicitly as an evolution equation for the number density $n_{\mathrm{E}}:=-n_{\mu} j^{\mu}=n \Gamma$ measured by the Eulerian observer as follows

$$
\partial_{t}\left(\sqrt{h} n_{\mathrm{E}}\right)+\partial_{i}\left[\sqrt{h} n_{\mathrm{E}} V^{i}\right]=0
$$

where $\Gamma$ and $V^{i}$ are given by Eqs. (44) and (46) respectively. Introducing the physical components of the fluid velocity field given by Eq. (45), we have the alternative expression,

$$
\partial_{t}\left(\sqrt{h} n_{\mathrm{E}}\right)+\partial_{i}\left[\sqrt{h} n_{\mathrm{E}}\left(N^{i}+N q_{(j)}^{i}{ }^{3} U^{(j)}\right)\right]=0
$$

The equation of conservation of baryon number leads to the conserved total baryon number given by

$$
\begin{aligned}
\mathcal{N} & =\int_{\Sigma_{t}}-j^{\mu} n_{\mu} \sqrt{h} d x^{1} d x^{2} d x^{3} \\
& =\int_{\Sigma_{t}} n \Gamma \sqrt{h} d x^{1} d x^{2} d x^{3}
\end{aligned}
$$

The integral has compact support corresponding to the volume enveloped by the star surface.

In a similar way, the equation for entropy conservation reads

$$
\nabla_{\mu}\left(s u^{\mu}\right)=-\frac{1}{\Theta}\left(\mu^{\mathrm{R}} \mathcal{R}_{\mathrm{R}}+\mathcal{D}\right)
$$

where

$$
\mathcal{R}_{\mathrm{R}}:=\nabla_{\mu}\left(n_{\mathrm{R}} u^{\mu}\right)
$$

is the rate of particle production and we remind that

$$
\mathcal{D}:=-u_{\nu} \nabla_{\mu} T_{\mathrm{R}}^{\mu \nu}=u_{\nu} \nabla_{\mu} T_{\mathrm{PF}}^{\mu \nu}
$$

is the particle's mean emissivity in the fluid frame. Therefore the sources for the entropy generation in a perfect fluid are from the particle production (e.g. neutrinos). The Eq. (118) is completely equivalent to Eq. (101) which is given in terms of the distribution function. 
One can define the entropy per baryon $\sigma=s / n$ and use Eqs. (112) and (117) to obtain

$$
u^{\mu} \nabla_{\mu} \sigma=-\frac{1}{n \Theta}\left(\mu^{\mathrm{R}} \mathcal{R}_{\mathrm{R}}+\mathcal{D}\right)
$$

Explicitly this provides an evolution equation for $\sigma$ :

$$
\partial_{t} \sigma+V^{i} \partial_{i} \sigma=-\frac{N}{n \Theta \Gamma}\left(\mu^{\mathrm{R}} \mathcal{R}_{\mathrm{R}}+\mathcal{D}\right)
$$

Moreover, using Eqs. (44), (45) and the tetrad approach of Sec. IIA, the Eq. (120) takes the alternative form:

$$
n^{\mu} \partial_{\mu} \sigma+{ }^{3} U^{(i)} \partial_{(i)} \sigma=-\frac{1}{n \Gamma \Theta}\left(\mu^{\mathrm{R}} \mathcal{R}_{\mathrm{R}}+\mathcal{D}\right)
$$

In the same way, we can introduce the particle number per baryon $x_{\mathrm{R}}=n_{\mathrm{R}} / n$ and write (118) as

$$
u^{\mu} \nabla_{\mu} x_{\mathrm{R}}=\frac{1}{n} \mathcal{R}_{\mathrm{R}}
$$

which provides an evolution equation for $x_{\mathrm{R}}$ :

$$
\partial_{t} x_{\mathrm{R}}+V^{i} \partial_{i} x_{\mathrm{R}}=\frac{N}{n \Gamma} \mathcal{R}_{\mathrm{R}}
$$

or alternatively,

$$
n^{\mu} \partial_{\mu} x_{\mathrm{R}}+{ }^{3} U^{(i)} \partial_{(i)} x_{\mathrm{R}}=\frac{1}{n \Gamma} \mathcal{R}_{\mathrm{R}}
$$

In order to close the whole system of equations presented so far one needs the input of particle physics. That is, the equation of state for nuclear matter, the rate of particle production and the opacities (see Ref. [2] for a review). In the case of neutrinos emitted by nuclear matter out of beta equilibrium via direct and inverse $\beta$ processes during neutron star collapse, the rate of particle production, the emissivities and the opacities can be given in terms of rather simple formulae [36,57] (see also Ref. [58] for neutrino emissivities from quark matter in $\beta$-equilibrium within neutron stars and Ref. [59] for neutrino emission from hot and dense atmospheres). For the case of reaction rates and opacities in Type II supernovae see for instance Ref. [15]. 


\section{SPHERICAL SYMMETRY}

In this and the following sections we will focus on spherically symmetric spacetimes. The most general line-element for such spacetimes according with the $3+1$ decomposition of the metric Eq. (3), writes

$$
d s^{2}=-\left(N^{2}-N^{r} N_{r}\right) d t^{2}-2 N_{r} d t d r+A^{2} d r^{2}+B^{2}\left(r^{2} d \theta^{2}+r^{2} \sin ^{2} \theta d \phi^{2}\right)
$$

where all the metric potentials are functions of the coordinates $r$ and $t$ solely. The three-metric $h_{i j}$ is easily read-off from (126).

On the other hand, the triad coefficients are

$$
e_{j}^{(i)}=\operatorname{diag}(A(r, t), r B(r, t), r \sin \theta B(r, t))
$$

The inverse coefficients $q_{(j)}^{i}$ can be obtained trivially form (127).

The extrinsic curvature can be computed from (4). We find [60],

$$
\begin{aligned}
& \left(K_{i j}\right)=\left(\begin{array}{ccc}
-\frac{A}{N}\left(\partial_{t} A+\partial_{r} N^{(r)}\right) & 0 & 0 \\
0 & -\frac{r B^{2}}{N}\left(\frac{N^{(r)}}{A}+\frac{r}{B} \partial_{t} B+\frac{r N^{(r)} \partial_{r} B}{A B}\right) & 0 \\
0 & 0 & -\frac{r B^{2} \sin ^{2}(\theta)}{N}\left(\frac{N^{(r)}}{A}+\frac{r}{B} \partial_{t} B+\frac{r N^{(r)} \partial_{r} B}{A B}\right)
\end{array}\right) \\
& \begin{aligned}
\left(K_{j}^{i}\right)=\left(K_{(j)}^{(i)}\right) & =\left(\begin{array}{ccc}
-\frac{1}{N}\left(\frac{\partial_{t} A}{A}+\frac{N^{r} \partial_{r} A}{A}+\partial_{r} N^{r}\right) & 0 & 0 \\
0 & -\frac{1}{N}\left(\frac{\partial_{t} B}{B}+\frac{N^{r} \partial_{r} B}{B}+\frac{N^{r}}{r}\right) & 0 \\
0 & 0 & -\frac{1}{N}\left(\frac{\partial_{t} B}{B}+\frac{N^{r} \partial_{r} B}{B}+\frac{N^{r}}{r}\right)
\end{array}\right) \\
& =\left(\begin{array}{ccc}
-\frac{1}{N}\left(\frac{\partial_{t} A}{A}+\frac{\partial_{r} N^{(r)}}{A}\right) & 0 & 0 \\
0 & -\frac{1}{N}\left(\frac{\partial_{t} B}{B}+\frac{N^{(r)} \partial_{r} B}{A B}+\frac{N^{(r)}}{r A}\right) & -\frac{1}{N}\left(\frac{\partial_{t} B}{B}+\frac{N^{(r)} \partial_{r} B}{A B}+\frac{N^{(r)}}{r A}\right)
\end{array}\right)
\end{aligned}
\end{aligned}
$$

where the index of Eq. (129) was raised with $h^{i j}$ from Eq. (128).

The three-scalar of curvature is given by

$$
{ }^{3} R=\frac{2}{r^{2} A^{2}}\left(\frac{A^{2}}{B^{2}}-1\right)+\frac{2}{A^{2}}\left(\frac{2 \partial_{r} A}{r A}+\frac{2\left(\partial_{r} B\right)\left(\partial_{r} A\right)}{B A}-\frac{\left(\partial_{r} B\right)^{2}}{B^{2}}-\frac{6 \partial_{r} B}{r B}-\frac{2 \partial_{r r}^{2} B}{B}\right) .
$$




\section{A. The $3+1$ Einstein equations}

It is useful to introduce the new variables,

$$
\begin{aligned}
& \nu:=\ln [N], \\
& \alpha:=\ln [A], \\
& \beta:=\ln [B] .
\end{aligned}
$$

The Hamiltonian constraint Eq.(10) reads

$$
\begin{aligned}
& \frac{1}{r^{2}}\left(\frac{A^{2}}{B^{2}}-1\right)+A^{2}\left[2 K_{(r)}^{(r)} K_{(\theta)}^{(\theta)}+\left(K_{(\theta)}^{(\theta)}\right)^{2}\right] \\
& +\frac{2 \partial_{r} \alpha}{r}-\frac{6 \partial_{r} \beta}{r}+2\left(\partial_{r} \alpha\right)\left(\partial_{r} \beta\right)-3\left(\partial_{r} \beta\right)^{2}-2 \partial_{r r}^{2} \beta=8 \pi G_{0} E A^{2} .
\end{aligned}
$$

where we have used the fact that the non-diagonal components of $K^{i}{ }_{j}$ are null and that $K_{(\theta)}^{(\theta)}=K_{(\phi)}^{(\phi)}[$ cf. Eq. 129$\left.)\right]$.

The radial component of the momentum constraints Eq.(11), reads

$$
\left(K_{(r)}^{(r)}-K_{(\theta)}^{(\theta)}\right)\left(\frac{1}{r}+\partial_{r} \beta\right)-\partial_{r} K_{(\theta)}^{(\theta)}=4 \pi G_{0} A J_{(r)}
$$

Or in terms of the trace $K$,

$$
2\left(K_{(r)}^{(r)}-K_{(\theta)}^{(\theta)}\right)\left(\frac{1}{r}+\partial_{r} \beta\right)-\partial_{r} K+\partial_{r} K_{(r)}^{(r)}=8 \pi G_{0} A J_{(r)}
$$

The angular components of Eq.(11) and the spherical symmetry lead to the conditions $J_{\theta}=0=J_{\phi}$ which implies the absence of "angular currents".

The dynamical equations for the non-diagonal components of Eq.(12), i.e., for $\partial_{t} K_{(\theta)}^{(r)}$, $\partial_{t} K_{(\phi)}^{(r)}, \partial_{t} K_{(\phi)}^{(\theta)}$ with the fact that $K_{(\theta)}^{(r)}=K_{(\phi)}^{(r)}=K_{(\phi)}^{(\theta)}=0$ [cf. Eq. (129)], leads respectively to the conditions that $S_{(\theta)}^{(r)}=S_{(\phi)}^{(r)}=S_{(\phi)}^{(\theta)}=0$. Moreover, taking into account the fact that $K_{(\theta)}^{(\theta)}=K_{(\phi)}^{(\phi)}$, the dynamical equations for $\partial_{t} K_{(\theta)}^{(\theta)}$ and $\partial_{t} K_{(\phi)}^{(\phi)}$ [see Eq. (12)],

leads to the condition $S_{(\theta)}^{(\theta)}=S_{(\phi)}^{(\phi)}$ corresponding to an "isotropic" energy-momentum tensor which is compatible with the hypothesis of a spacetime with spherical symmetry. In this way, the only two non-trivial dynamical equations are 


$$
\begin{gathered}
\partial_{t} K_{(r)}^{(r)}+\frac{N^{(r)} \partial_{r} K_{(r)}^{(r)}}{A}-N K K_{(r)}^{(r)}-\frac{N}{A^{2}}\left(\frac{2 \partial_{r} \alpha}{r}-\frac{4 \partial_{r} \beta}{r}+2\left(\partial_{r} \alpha\right)\left(\partial_{r} \beta\right)-2\left(\partial_{r} \beta\right)^{2}-2 \partial_{r r}^{2} \beta\right) \\
+\frac{N}{A^{2}}\left[\partial_{r r}^{2} \nu+\left(\partial_{r} \nu\right)^{2}-\left(\partial_{r} \alpha\right)\left(\partial_{r} \nu\right)\right]=4 \pi G_{0} N\left(-S_{(r)}^{(r)}+2 S_{(\theta)}^{(\theta)}-E\right), \\
\partial_{t} K_{(\theta)}^{(\theta)}+\frac{N^{(r)} \partial_{r} K_{(\theta)}^{(\theta)}-N K K_{(\theta)}^{(\theta)}-\frac{N}{A^{2}}\left[\frac{1}{r^{2}}\left(\frac{A^{2}}{B^{2}}-1\right)+\frac{\partial_{r} \alpha}{r}-\frac{4 \partial_{r} \beta}{r}+\left(\partial_{r} \alpha\right)\left(\partial_{r} \beta\right)-2\left(\partial_{r} \beta\right)^{2}-\partial_{r r}^{2} \beta\right]}{+\frac{N}{A^{2}}\left[\left(\partial_{r} \beta\right)\left(\partial_{r} \nu\right)+\frac{\partial_{r} \nu}{r}\right]=4 \pi G_{0} N\left(S_{(r)}^{(r)}-E\right) .}
\end{gathered}
$$

From Eq. (14) the evolution equation for the trace of $K_{i j}$ is

$$
\begin{aligned}
& \partial_{t} K+\frac{N^{(r)} \partial_{r} K}{A}-N\left[\left(K_{(r)}^{(r)}\right)^{2}+2\left(K_{(\theta)}^{(\theta)}\right)^{2}\right] \\
& +\frac{N}{A^{2}}\left[\partial_{r r}^{2} \nu+\left(\partial_{r} \nu\right)^{2}-\left(\partial_{r} \alpha\right)\left(\partial_{r} \nu\right)+2\left(\partial_{r} \beta\right)\left(\partial_{r} \nu\right)+\frac{2 \partial_{r} \nu}{r}\right]=4 \pi G_{0} N(S+E)
\end{aligned}
$$

where we recognize the 3-covariant Laplace operator in spherical coordinates of the slices $\Sigma_{t}[$ see Eq. (126)]:

$$
{ }^{3} \Delta \nu=\frac{1}{A^{2}}\left[\partial_{r r}^{2} \nu+\frac{2 \partial_{r} \nu}{r}-\left(\partial_{r} \alpha\right)\left(\partial_{r} \nu\right)+2\left(\partial_{r} \beta\right)\left(\partial_{r} \nu\right)\right]
$$

\section{B. Matter equations}

In the present case of a perfect fluid in spherical symmetry we have

$$
\begin{aligned}
E_{\mathrm{PF}} & =(\rho+p) \Gamma-p, \\
\mathrm{PF}_{(r)}^{(r)} & =\left(E_{\mathrm{PF}}+p\right)\left({ }^{3} U^{(r)}\right)^{2}+p, \\
\mathrm{PF}_{(\theta)}^{(\theta)} & =S_{(\phi)}^{(\phi)}=p \\
J_{(r)}^{\mathrm{PF}} & =\left(E_{\mathrm{PF}}+p\right){ }^{3} U^{(r)}, \\
\Gamma & =\left[1-\left({ }^{3} U^{(r)}\right)^{2}\right]^{-1 / 2},
\end{aligned}
$$

where

$$
{ }^{3} U^{(r)}=\frac{A}{N}\left(V^{r}-N^{r}\right)=\frac{1}{N}\left(V^{(r)}-N^{(r)}\right),
$$


with $V^{r}:=u^{r} / u^{t}$. The spherical symmetry implies $u^{\theta}=0=u^{\phi}$ and therefore $U^{(\theta)}=0=U^{(\phi)}$.

Note that

$$
\mathrm{PF} S_{(r)}^{(r)}={ }^{3} U^{(r)} J_{(r)}^{\mathrm{PF}}+p
$$

The Eq. (47) reads

$\partial_{t} E_{\mathrm{PF}}+N^{r} \partial_{r} E_{\mathrm{PF}}+\frac{N}{A B^{2} r^{2}} \partial_{r}\left(A B^{2} r^{2} J_{\mathrm{PF}}^{r}\right)=N\left({ }_{\mathrm{PF}} S_{(r)}^{(r)} K_{(r)}^{(r)}+2{ }_{\mathrm{PF}} S_{(\theta)}^{(\theta)} K_{(\theta)}^{(\theta)}+E_{\mathrm{PF}} K\right)-2 J_{\mathrm{PF}}^{r} \partial_{r} N-N^{2} \mathcal{F}^{t}$

Or in terms of $J_{(r)}=A J^{r}$,

$\partial_{t} E_{\mathrm{PF}}+N^{r} \partial_{r} E_{\mathrm{PF}}+\frac{N}{A B^{2} r^{2}} \partial_{r}\left(B^{2} r^{2} J_{(r)}^{\mathrm{PF}}\right)=N\left({ }_{\mathrm{PF}} S_{(r)}^{(r)} K_{(r)}^{(r)}+2{ }_{\mathrm{PF}} S_{(\theta)}^{(\theta)} K_{(\theta)}^{(\theta)}+E_{\mathrm{PF}} K\right)-\frac{2}{A} J_{(r)}^{\mathrm{PF}} \partial_{r} N-N^{2} \mathcal{F}^{t}$.

Using Eqs. (143), (146) and (147) the latter equation can be written in conservative form as

$$
\begin{aligned}
\partial_{t} E_{\mathrm{PF}}+\frac{1}{r^{2}} \partial_{r}\left(r^{2} V^{r} E_{\mathrm{PF}}\right)= & E_{\mathrm{PF}}\left(N K+\frac{2 N^{r}}{r}+\partial_{r} N^{r}\right)-\frac{1}{r^{2}} \partial_{r}\left(r^{2} \frac{N}{A}{ }^{3} U^{(r)} p\right) \\
& -\frac{N J_{(r)}^{\mathrm{PF}}}{A}\left[\partial_{r} \nu+\partial_{r} \alpha+2 \partial_{r} \beta-{ }^{3} U^{(r)} A K_{(r)}^{(r)}\right]+N K p-N^{2} \mathcal{F}^{t} .
\end{aligned}
$$

The momentum conservation Eq.(19) reads

$$
\partial_{t} J_{r}^{\mathrm{PF}}+N^{r} \partial_{r} J_{r}^{\mathrm{PF}}+J_{r}^{\mathrm{PF}} \partial_{r} N^{r}+N\left({ }^{3} \nabla_{l \mathrm{PF}} S_{r}^{l}\right)=N K J_{r}^{\mathrm{PF}}-\left({ }_{\mathrm{PF}} S_{r}^{r}+E_{\mathrm{PF}}\right) \partial_{r} N-{ }^{3} \mathcal{F}_{r} N
$$

Explicitly

$$
\begin{aligned}
& \partial_{t} J_{r}^{\mathrm{PF}}+N^{r} \partial_{r} J_{r}^{\mathrm{PF}}+J_{r}^{\mathrm{PF}} \partial_{r} N^{r}+N \partial_{r}{ }_{\mathrm{PF}} S_{(r)}^{(r)}+2 N\left(\mathrm{PF}_{(r)}^{(r)}-\mathrm{PF}_{(\theta)} S_{(\theta)}^{(\theta)}\right)\left(\frac{1}{r}+\frac{\partial_{r} B}{B}\right) \\
& =-\left({ }_{\mathrm{PF}} S_{(r)}^{(r)}+E_{\mathrm{PF}}\right) \partial_{r} N+N J_{r}^{\mathrm{PF}}\left(K_{(r)}^{(r)}+2 K_{(\theta)}^{(\theta)}\right)-{ }^{3} \mathcal{F}_{r} N .
\end{aligned}
$$

Or in terms of $J_{(r)}$, we have

$$
\begin{aligned}
& \partial_{t} J_{(r)}^{\mathrm{PF}}+J_{(r)}^{\mathrm{PF}} \frac{\partial_{t} A}{A}+\frac{N^{(r)}}{A} \partial_{r} J_{(r)}^{\mathrm{PF}}+\frac{J_{(r)}^{\mathrm{PF}}}{A} \partial_{r} N^{(r)}+\frac{N}{A} \partial_{r \mathrm{PF}} S_{(r)}^{(r)}+\frac{2 N}{A}\left({ }_{\mathrm{PF}} S_{(r)}^{(r)}-{ }_{\mathrm{PF}} S_{(\theta)}^{(\theta)}\right)\left(\frac{1}{r}+\frac{\partial_{r} B}{B}\right) \\
& =-\left(\mathrm{PF}_{(r)}^{(r)}+E_{\mathrm{PF}}\right) \frac{\partial_{r} N}{A}+N J_{(r)}^{\mathrm{PF}}\left(K_{(r)}^{(r)}+2 K_{(\theta)}^{(\theta)}\right)-{ }^{3} \mathcal{F}_{(r)} N .
\end{aligned}
$$


When using Eq. (129) to replace the time derivative of $A$ we find

$$
\begin{aligned}
& \partial_{t} J_{(r)}^{\mathrm{PF}}+\frac{N^{(r)}}{A} \partial_{r} J_{(r)}^{\mathrm{PF}}+\frac{N}{A} \partial_{r \mathrm{PF}} S_{(r)}^{(r)}+\frac{2 N}{A}\left({ }_{\mathrm{PF}} S_{(r)}^{(r)}-{ }_{\mathrm{PF}} S_{(\theta)}^{(\theta)}\right)\left(\frac{1}{r}+\frac{\partial_{r} B}{B}\right) \\
& =-\left({ }_{\mathrm{PF}} S_{(r)}^{(r)}+E_{\mathrm{PF}}\right) \frac{\partial_{r} N}{A}+2 N J_{(r)}^{\mathrm{PF}}\left(K_{(r)}^{(r)}+K_{(\theta)}^{(\theta)}\right)-{ }^{3} \mathcal{F}_{(r)} N .
\end{aligned}
$$

Using Eqs. (146) and (147), one obtains an equation for $J_{(r)}^{\mathrm{PF}}$ in conservative form

$$
\begin{aligned}
\partial_{t} J_{(r)}^{\mathrm{PF}}+\frac{1}{r^{2}} \partial_{r}\left(r^{2} V^{r} J_{(r)}^{\mathrm{PF}}\right)= & \frac{N}{A}\left\{J_{(r)}^{\mathrm{PF}}\left[2 A\left(K_{(r)}^{(r)}+K_{(\theta)}^{(\theta)}\right)-{ }^{3} U^{(r)}\left(\partial_{r} \alpha+2 \partial_{r} \beta\right)-\frac{A}{N}\left(\frac{2}{r} N^{r}+\partial_{r} N^{r}\right)\right]\right. \\
& \left.-\left(E_{\mathrm{PF}}+p\right) \partial_{r} \nu-\partial_{r} p-{ }^{3} \mathcal{F}_{(r)} A\right\} .
\end{aligned}
$$

with the alternative form for the r.h.s,

$$
\begin{aligned}
\partial_{t} J_{(r)}^{\mathrm{PF}}+\frac{1}{r^{2}} \partial_{r}\left(r^{2} V^{r} J_{(r)}^{\mathrm{PF}}\right)= & J_{(r)}^{\mathrm{PF}}\left[2 N\left(K_{(r)}^{(r)}+K_{(\theta)}^{(\theta)}\right)-V^{r}\left(\partial_{r} \alpha+2 \partial_{r} \beta\right)+N^{r}\left(\partial_{r} \alpha+2 \partial_{r} \beta-\frac{2}{r}\right)-\partial_{r} N^{r}\right] \\
& -\frac{N}{A}\left[\left(E_{\mathrm{PF}}+p\right) \partial_{r} \nu+\partial_{r} p+{ }^{3} \mathcal{F}_{(r)} A\right] .
\end{aligned}
$$

Another possibility which has turned to be very useful in some numerical studies [8, 9, 36], is the use of the Euler equation for the fluid instead of the equation for $J_{(r)}^{\mathrm{PF}}$. The only non-trivial component of the Euler equation (48) in spherical symmetry reads

$$
\begin{aligned}
& \partial_{(t)}{ }^{3} U^{(r)}+{ }^{3} U^{(r)}{ }^{3} \partial_{(r)}{ }^{3} U^{(r)}=-\frac{1}{E_{\mathrm{PF}}+p}\left[{ }^{3} \partial^{(r)} p+{ }^{3} U^{(r)} \partial_{(t)} p\right] \\
+ & \frac{1}{\Gamma^{2}}\left({ }^{3} U^{(r)} K_{(r)}^{(r)}-a_{(r)}\right)+\frac{1}{E_{\mathrm{PF}}+p}\left({ }^{3} U^{(r)} \mathcal{F}^{(t)}-\mathcal{F}^{(r)}\right) .
\end{aligned}
$$

where we used the fact that the RRC are antisymmetric so that the terms $\mathcal{O}_{(t)(r)}^{(r)}=0=\mathcal{O}_{(r)(r)}^{(r)}$ and we remind the explicit expressions

$$
\begin{aligned}
\partial_{(t)} & =\frac{1}{N} \partial_{t}+\frac{N^{r}}{N} \partial_{r}, \\
{ }^{3} \partial_{(r)} & =\frac{1}{A} \partial_{r} \\
a_{(r)} & ={ }^{3} \partial_{(r)} \nu .
\end{aligned}
$$




\section{The RBE in spherical symmetry}

The most general 4-metric for a spherically symmetric spacetime is given by Eq.(126). Then, it's easy to see that the simplest tetrad choice $e_{(\mu)}$ associated to Eq.(126) and which corresponds to the local tetrad of the Eulerian observer, reads,

$$
\begin{aligned}
e_{(t)} & =\frac{1}{N} \frac{\partial}{\partial t}+\frac{N^{r}}{N} \frac{\partial}{\partial r} \\
e_{(r)} & =\frac{1}{A} \frac{\partial}{\partial r} \\
e_{(\theta)} & =\frac{1}{r B} \frac{\partial}{\partial \theta} \\
e_{(\phi)} & =\frac{1}{r B \sin \theta} \frac{\partial}{\partial \phi}
\end{aligned}
$$

where $\partial / \partial x^{\mu}\left[\right.$ with $\left.x^{\mu}=(t, r, \theta, \phi)\right]$ denote the coordinate basis.

In the Eulerain frame, the spherical symmetry of configuration space (i.e., spacetime) induces a symmetry on momentum space that can be exploited to simplify the computations. It is convenient to define spherical variables on momentum space as follows: take a unit vector $e_{(r)}$ as polar axis, i.e., as symmetry axis on momentum space. Then it is useful to introduce new variables $e, \psi, \gamma$ in the Eulerian frame as

$$
p^{(t)}=e, p^{(r)}=p \cos \psi, p^{(\theta)}=p \sin \psi \cos \gamma, p^{(\phi)}=p \sin \psi \sin \gamma
$$

As mentioned earlier in Sec. IV, $e$ is the energy of the radiated particles (e.g., neutrinos) in

the Eulerian frame and $p^{2}=p^{(i)} p_{(i)} ; \psi$ is the angle between the polar axis and the neutrino propagation three-vector $p^{(i)}$, and $\gamma$ is the angle of rotation around $e_{(r)}$. The above variables are consitent with the mass shell condition (61). For massless particles, then $p \equiv e$. Under these new variables the $\mathrm{RBE}(83)$ reads

$$
\left(p^{(a)}(e, \psi, \gamma) q_{(a)}^{\mu} \frac{\partial}{\partial x^{\mu}}-p^{(b)}(e, \psi, \gamma) p^{(a)}(e, \psi, \gamma) \mathcal{O}_{(b)(a)}^{(d)} \frac{\partial \tilde{p}^{(c)}}{\partial p^{(d)}} \frac{\partial}{\partial \tilde{p}^{(c)}}\right) F(\tilde{\boldsymbol{p}})=\left(\frac{d F}{d \tau}\right)_{\mathrm{coll}}
$$

where we have explicitly stress the dependence of momenta with respect to the new momenta spherical-like variables $(e, p, \psi, \gamma)$ represented colectively by $\tilde{\boldsymbol{p}}$. 
Indeed, the spherical symmetry and the mass shell condition will be reflected in the RBE by the fact that the distribution function $F_{\mathrm{R}}$ depends only of four phase-space coordinates $(t, r, e, \mu)$ or $(t, r, p, \mu)$ instead of the original eight $\left(x^{\mu}, p^{\mu}\right)$ [56, 61, where $\mu:=\cos \psi$.

We can now proceed to calculate explicitly the RBE. The only non-null Ricci coefficients are [60],

$$
\begin{aligned}
\mathcal{O}_{(t)(r)}^{(t)} & =\mathcal{O}_{(t)(t)}^{(r)}=D_{r} \nu, \mathcal{O}_{(r)(r)}^{(t)}=\mathcal{O}_{(r)(t)}^{(r)}=D_{t} \alpha+\frac{1}{N} D_{r} N^{(r)}, \\
\mathcal{O}_{(\theta)(\theta)}^{(t)} & =\mathcal{O}_{(\phi)(\phi)}^{(t)}=\mathcal{O}_{(\theta)(t)}^{(\theta)}=\mathcal{O}_{(\phi)(t)}^{(\phi)}=\frac{N^{(r)}}{r A N}+D_{t} \beta+\frac{N^{(r)}}{N} D_{r} \beta, \\
\mathcal{O}_{(\theta)(\theta)}^{(r)} & =\mathcal{O}_{(\phi)(\phi)}^{(r)}=-\mathcal{O}_{(\theta)(r)}^{(\theta)}=-\mathcal{O}_{(\phi)(r)}^{(\phi)}=-r^{-1} A^{-1}-D_{r} \beta, \\
\mathcal{O}_{(\phi)(\phi)}^{(\theta)} & =-\mathcal{O}_{(\phi)(\theta)}^{(\phi)}=-\frac{1}{r B} \cot \theta,
\end{aligned}
$$

where $D_{t}:=\frac{1}{N} \frac{\partial}{\partial t}, D_{r}:=\frac{1}{A} \frac{\partial}{\partial r} \equiv{ }^{3} \partial_{(r)}$. After imposing the mass shell condition, the RBE (166) in terms of the spherical variables reads explicilty [60],

$$
\begin{aligned}
& e D_{t} F_{\mathrm{R}}(r, t, e, \mu)+\left(\frac{p \mu}{e}+\frac{N^{(r)}}{N}\right) e D_{r} F_{\mathrm{R}}(r, t, e, \mu) \\
& -p^{2}\left[\left(1-\mu^{2}\right) \frac{N^{(r)}}{N}\left(\frac{1}{r A}+D_{r} \beta\right)+\mu^{2} D_{t} \alpha+\left(1-\mu^{2}\right) D_{t} \beta+\frac{\mu e}{p} D_{r} \nu+\frac{\mu^{2}}{N} D_{r} N^{(r)}\right] \partial_{e} F_{\mathrm{R}}(r, t, e, \mu) \\
& +e\left(1-\mu^{2}\right)\left[\left(\frac{p}{e}+\frac{\mu N^{(r)}}{N}\right)\left(\frac{1}{r A}+D_{r} \beta\right)+\mu\left(D_{t} \beta-D_{t} \alpha\right)-\frac{e}{p} D_{r} \nu-\frac{\mu}{N} D_{r} N^{(r)}\right] \partial_{\mu} F_{\mathrm{R}}(r, t, e, \mu) \\
& =\left(\frac{d F}{d \lambda}\right)_{\text {coll }} .
\end{aligned}
$$

The alternative $3+1$ form of the RBE can be computed from (87) when changing to the spherical variables $\tilde{p}^{(\mu)}$ in the momentum space:

$$
\begin{aligned}
& e D_{t} F_{\mathrm{R}}(r, t, E, \mu)+\left(\frac{p \mu}{e}+\frac{N^{(r)}}{N}\right) e D_{r} F_{\mathrm{R}}(r, t, e, \mu)-p^{2}\left[-\left(1-\mu^{2}\right) K_{(\theta)}^{(\theta)}-\mu^{2} K_{(r)}^{(r)}+\frac{\mu e}{p} D_{r} \nu\right] \partial_{e} F_{\mathrm{R}}(r, t, e, \mu) \\
& +e\left(1-\mu^{2}\right)\left[\frac{p}{e}\left(\frac{1}{r A}+D_{r} \beta\right)+\mu\left(K_{(r)}^{(r)}-K_{(\theta)}^{(\theta)}\right)-\frac{e}{p} D_{r} \nu\right] \partial_{\mu} F_{\mathrm{R}}(r, t, e, \mu)=\left(\frac{d F}{d \lambda}\right)_{\text {coll }} .
\end{aligned}
$$

In normal coordinates where $N^{r}=0$, and for massless particles, Eq.(166) reduces to the relativistic Boltzman equation derived by Lindquist [56] with the choice $B=R(r) / r$. Under the isotropic gauge Eq. (168) can be written in a "conservative" form which is specially suited 
for numerical solutions [45]. Following Harleston \& Vishniac [44, we can write Eq.(168) in conservative form as

$$
\partial_{t} \tilde{F}+\frac{1}{r^{2}} \partial_{r}\left(r^{2} V_{p}^{r} \tilde{F}\right)+\frac{1}{p e} \partial_{e}\left(p^{3} \mathcal{H}_{e} \tilde{F}\right)+\partial_{\mu}\left(\mathcal{H}_{\mu} \tilde{F}\right)=\frac{N A B^{2}}{e}\left(\frac{d F}{d \lambda}\right)_{\text {coll }}
$$

where

$$
\begin{aligned}
\tilde{F} & =A B^{2} F_{\mathrm{R}}(r, t, e, \mu) \\
V_{p}^{r} & =\frac{p^{r}}{p^{t}}=\frac{N}{A}\left(\frac{p \mu}{e}+\frac{N^{(r)}}{N}\right), \\
\mathcal{H}_{e} & =\frac{N}{p^{2}} \hat{\boldsymbol{L}}(e)=-N\left[-\left(1-\mu^{2}\right) K_{(\theta)}^{(\theta)}-\mu^{2} K_{(r)}^{(r)}+\frac{\mu e}{p} D_{r} \nu\right] \\
\mathcal{H}_{\mu} & =\frac{N}{e} \hat{\boldsymbol{L}}(\mu)=N\left(1-\mu^{2}\right)\left[\frac{p}{e}\left(\frac{1}{r A}+D_{r} \beta\right)+\mu\left(K_{(r)}^{(r)}-K_{(\theta)}^{(\theta)}\right)-\frac{e}{p} D_{r} \nu\right],
\end{aligned}
$$

and $\hat{\boldsymbol{L}}$ stands for the Liouville operator as it appears in the l.h.s of Eq.(168). Alternatively, the above equation can be written as

$$
\partial_{t} \tilde{F}+\frac{1}{r^{2}} \partial_{r}\left(r^{2} V_{p}^{r} \tilde{F}\right)+\frac{1}{p^{2}} \partial_{p}\left(p^{3} \mathcal{H}_{p} \tilde{F}\right)+\partial_{\mu}\left(\mathcal{H}_{\mu} \tilde{F}\right)=\frac{N A B^{2}}{e}\left(\frac{d F}{d \lambda}\right)_{\text {coll }},
$$

where now $\tilde{F}=\tilde{F}_{\mathrm{R}}(r, t, p, \mu)$ is to be regarded as a function of $p$ instead of $e$ and

$$
\mathcal{H}_{p}=\frac{N}{p e} \hat{\boldsymbol{L}}(p)=-N\left[-\left(1-\mu^{2}\right) K_{(\theta)}^{(\theta)}-\mu^{2} K_{(r)}^{(r)}+\frac{\mu e}{p} D_{r} \nu\right]
$$

When one of the different gauges and slicing conditions are chosen to write the RBE this will only affect the particular form of the quantities given by $(170)-(173)$ and $(175)$, and the r.h.s of Eqs.(169) and (174).

Finally, we emphasize that all the momentum variables which appear in the various forms of the RBE in spherical symmetry, are components with respect to the orthonormal tetrad carried by the Eulerian observer. Therefore, the corresponding quantities in the collision integral are to be referred to the same observer. 


\section{Mean radiative variables}

In Sec. IV we defined the energy-momentum tensor of particles in terms of their microscopic four-momenta. However, we can introduce mean radiative variables which are to be interpreted as their counterparts of the continuum case. Such variables are called the moments of the distribution function. In order to write them explicitly, we shall use the physical components of the energy-momentum tensor of particles Eq. (67) and the spherical variables in momentum space in addition to the invariant volume element of momentum space given by Eq.(65). We have then

$$
\begin{aligned}
T_{\mathrm{R}}^{(\mu)(\nu)} & =\int_{0}^{\infty} \int_{-1}^{1} \int_{0}^{2 \pi} p^{(\mu)} p^{(\nu)} F_{\mathrm{R}}(r, t, p, \mu) \frac{p^{2}}{e} d p d \mu d \gamma \\
& =\int_{\tilde{m}}^{\infty} \int_{-1}^{1} \int_{0}^{2 \pi} p^{(\mu)} p^{(\nu)} F_{\mathrm{R}}(r, t, e, \mu) \sqrt{e^{2}-\tilde{m}^{2}} d e d \mu d \gamma .
\end{aligned}
$$

The only non-null moments are

$$
\begin{aligned}
& E_{\mathrm{R}}:=T_{\mathrm{R}}^{(t)(t)}=2 \pi \int_{0}^{\infty} p^{2} \sqrt{p^{2}+\tilde{m}^{2}} d p \int_{-1}^{1} F_{\mathrm{R}}(r, t, p, \mu) d \mu=2 \pi \int_{\tilde{m}}^{\infty} e^{2} \sqrt{e^{2}-\tilde{m}^{2}} d e \int_{-1}^{1} F_{\mathrm{R}}(r, t, e, \mu) d \mu \\
& H_{\mathrm{R}}:=T_{\mathrm{R}}^{(t)(r)}=2 \pi \int_{0}^{\infty} p^{3} d p \int_{-1}^{1} \mu F_{\mathrm{R}}(r, t, e, \mu) d \mu=2 \pi \int_{\tilde{m}}^{\infty} e\left(e^{2}-\tilde{m}^{2}\right) d e \int_{-1}^{1} \mu F_{\mathrm{R}}(r, t, e, \mu) d \mu \\
& p_{\mathrm{R}}:=T_{\mathrm{R}}^{(r)(r)}=2 \pi \int_{0}^{\infty} \frac{p^{4}}{\sqrt{p^{2}+\tilde{m}^{2}}} d p \int_{-1}^{1} \mu^{2} F_{\mathrm{R}}(r, t, e, \mu) d \mu=2 \pi \int_{\tilde{m}}^{\infty}\left(e^{2}-\tilde{m}^{2}\right)^{3 / 2} d e \int_{-1}^{1} \mu^{2} F_{\mathrm{R}}(r, t, e, \mu) d \mu
\end{aligned}
$$

The tangential preassures are given by,

$$
\begin{aligned}
p_{\mathrm{R}}^{T}:=T_{\mathrm{R}}^{(\theta)(\theta)}=T_{\mathrm{R}}^{(\phi)(\phi)} & =\pi \int_{0}^{\infty} \frac{p^{4}}{\sqrt{p^{2}+\tilde{m}^{2}}} d p \int_{-1}^{1}\left(1-\mu^{2}\right) F_{\mathrm{R}}(r, t, e, \mu) d \mu \\
& =\pi \int_{\tilde{m}}^{\infty}\left(e^{2}-\tilde{m}^{2}\right)^{3 / 2} d e \int_{-1}^{1}\left(1-\mu^{2}\right) F_{\mathrm{R}}(r, t, e, \mu) d \mu
\end{aligned}
$$

We note that in the massless case,

$$
p_{\mathrm{R}}^{T}=\frac{1}{2}\left(E_{\mathrm{R}}-p_{\mathrm{R}}\right)
$$

The corresponding $3+1$ variables are 


$$
\begin{aligned}
J_{\mathrm{R}}^{(r)} & =H_{\mathrm{R}}, \\
S_{\mathrm{R}}^{(r)(r)} & =p_{\mathrm{R}}, \\
S_{\mathrm{R}}^{(\theta)(\theta)} & =S_{\mathrm{R}}^{(\phi)(\phi)}=p_{\mathrm{R}}^{T}, \\
S_{\mathrm{R}} & ={ }_{\mathrm{R}} S^{(i)}{ }_{(i)}^{(i)}=p_{\mathrm{R}}+2 p_{\mathrm{R}}^{T} .
\end{aligned}
$$

The effective pressure of radiation which can be defined as $p_{\text {eff }}^{\mathrm{R}}=S_{\mathrm{R}} / 3$ turns to be in the case of massless particles $p_{\mathrm{eff}}^{\mathrm{R}}=E_{\mathrm{R}} / 3$ which corresponds precisely to the equation of state (EOS) of an ultrarelativistic gas. In Eq. (178), $H_{\mathrm{R}}$ is the mean radiative flux of energy in the radial direction.

It is usual to introduce the so called variable Eddington factor

$$
\Xi=\frac{p_{\mathrm{R}}}{E_{\mathrm{R}}}
$$

used to measure the degree of "anisotropy" in the particle flow. In the case of massless particles if $\Xi=1 / 3$ then $p_{\mathrm{R}}^{T}=p_{\mathrm{R}}=E_{\mathrm{R}} / 3$ which corresponds to an fully isotropic flow. Moreover, in the free streaming approximation we have

$$
E_{\mathrm{R}}=J_{\mathrm{R}}^{(r)}=S_{\mathrm{R}}^{(r)(r)},
$$

and so $\Xi=1$, which is the case of a highly anisotropic flow $\left(p_{\mathrm{R}}^{T}=0\right)$ with a purely radial flux of radiation.

In the same way, the macroscopic particle number density current measured in the Eulerian frame is given by Eq. (66) and it in terms of the spherical variables of momentum space gives

$$
\begin{aligned}
j_{\mathrm{R}}^{(\mu)} & =\int_{0}^{\infty} \int_{-1}^{1} \int_{0}^{2 \pi} p^{(\mu)} F_{\mathrm{R}}(r, t, p, \mu) \frac{p^{2}}{e} d p d \mu d \gamma \\
& =\int_{\tilde{m}}^{\infty} \int_{-1}^{1} \int_{0}^{2 \pi} p^{(\mu)} F_{\mathrm{R}}(r, t, e, \mu) \sqrt{e^{2}-\tilde{m}^{2}} d e d \mu d \gamma
\end{aligned}
$$

In particular, the mean number density and flux of particles measured by the Eulerian observer are given respectively by

$$
n_{\mathrm{E}}^{\mathrm{R}}:=-n_{\mu} j_{\mathrm{R}}^{\mu}=j_{\mathrm{R}}^{(t)}=2 \pi \int_{0}^{\infty} \int_{-1}^{1} F_{\mathrm{R}}(r, t, p, \mu) p^{2} d p d \mu
$$




$$
\begin{aligned}
& \quad=2 \pi \int_{\tilde{m}}^{\infty} \int_{-1}^{1} \int_{0}^{2 \pi} e F_{\mathrm{R}}(r, t, e, \mu) \sqrt{e^{2}-\tilde{m}^{2}} d e d \mu \\
& j_{\mathrm{R}}^{(r)}=2 \pi \int_{0}^{\infty} \frac{p^{3}}{\sqrt{p^{2}+\tilde{m}^{2}}} d p \int_{-1}^{1} \mu F_{\mathrm{R}}(r, t, e, \mu) d \mu=2 \pi \int_{\tilde{m}}^{\infty}\left(e^{2}-\tilde{m}^{2}\right) d e \int_{-1}^{1} \mu F_{\mathrm{R}}(r, t, e, \mu) d \mu \\
& \text { and } j_{\mathrm{R}}^{(\theta)}=0=j_{\mathrm{R}}^{(\phi)} .
\end{aligned}
$$

In terms of the above macroscopic variables, the energy-momentum conservation equation in spherical symmetry Eq. (100) reads according to Eqs. (36) and (37) as follows 60]:

$$
\begin{gathered}
\partial_{(t)} E_{\mathrm{R}}+{ }^{3} \partial_{(r)} H_{\mathrm{R}}-E_{\mathrm{R}} K+2 H_{\mathrm{R}}\left[{ }^{3} \partial_{(r)} \nu+\frac{1}{r A}+{ }^{3} \partial_{(r)} \beta\right]-p_{\mathrm{R}} K_{(r)(r)}=-\mathcal{F}^{(t)} . \\
\partial_{(t)} H_{\mathrm{R}}+{ }^{3} \partial_{(r)} p_{\mathrm{R}}+\left[\frac{1}{r A}+\partial_{(r)} \beta\right]\left[3 p_{\mathrm{R}}-E_{\mathrm{R}}\right]+\left[p_{\mathrm{R}}+E_{\mathrm{R}}\right]^{3} \partial_{(r)} \nu-H_{\mathrm{R}}\left(K+K_{(r)}^{(r)}\right)=-\mathcal{F}^{(i)} .
\end{gathered}
$$

Depeding on the gauge and slicing choice some of the terms within these equations can vanish. Actually such evolution equations can be obtained directly from the RBE when multiplying this by the momenta and then integrating in momentum space.

We emphasize that it is more convenient to calculate $E_{\mathrm{R}}, H_{\mathrm{R}}$ and $p_{\mathrm{R}}$, directly from their definition once the distribution function has been computed, rather than using the above equations. In any case, such conservation equations can be used to verify the self-consistence of the system. The disadvantage of using the system of Eqs. (191) and (192) for the moments of the distribution instead of solving the RBE is that such a system is undetermined (i.e, there are more variables than equations). Then a closure relation is needed to remove the ambiguity (e.g. the diffusion approximation relating $E_{\mathrm{R}}$ and $p_{\mathrm{R}}$ ).

Finally, in spherical symmetry the evolution equations (113), (114), (121), (122), (124), (125) write respectively

$$
\begin{gathered}
\partial_{t}\left(A B^{2} n_{E}\right)+\frac{1}{r^{2}} \partial_{r}\left(r^{2} V^{r} n_{E} A B^{2}\right)=0 \\
\partial_{t}\left(A B^{2} n_{E}\right)+\frac{1}{r^{2}} \partial_{r}\left[A B^{2} r^{2} n_{E}\left(N^{r}+\frac{N}{A}{ }^{3} U^{(r)}\right)\right]=0 \\
\partial_{t} \sigma+V^{r} \partial_{r} \sigma=-\frac{N}{n \Theta \Gamma}\left(\mu^{\mathrm{R}} \mathcal{R}_{\mathrm{R}}+\mathcal{D}_{p}\right)
\end{gathered}
$$




$$
\begin{gathered}
\partial_{t} \sigma+N^{r} \partial_{r} \sigma+\frac{N^{3} U^{(r)}}{A} \partial_{r} \sigma=-\frac{N}{n \Gamma \Theta}\left(\mu^{\mathrm{R}} \mathcal{R}_{\mathrm{R}}+\mathcal{D}_{p}\right) \\
\partial_{t} x_{\mathrm{R}}+V^{r} \partial_{r} x_{\mathrm{R}}=\frac{N}{n \Gamma} \mathcal{R}_{\mathrm{R}} \\
\partial_{t} x_{\mathrm{R}}+N^{r} \partial_{r} x_{\mathrm{R}}+\frac{N^{3} U^{(r)}}{A} \partial_{r} x_{\mathrm{R}}=\frac{N}{n \Gamma} \mathcal{R}_{\mathrm{R}}
\end{gathered}
$$

Equation (193) has a conservative form for the quantity $A B^{2} n_{E}$. One can alternatively write an equation in conservative form for $n_{E}$ as

$$
\partial_{t} n_{E}+\frac{1}{r^{2}} \partial_{r}\left(r^{2} V^{r} n_{E}\right)+n_{E}\left[\partial_{t} \alpha+2 \partial_{t} \beta+V^{r}\left(\partial_{r} \alpha+2 \partial_{r} \beta\right)\right]=0
$$

In a similar way,

$$
\partial_{t} n_{E}+\frac{1}{r^{2}} \partial_{r}\left[r^{2} n_{E}\left(N^{r}+\frac{N}{A}{ }^{3} U^{(r)}\right)\right]+n_{E}\left[\partial_{t} \alpha+2 \partial_{t} \beta+\left(N^{r}+\frac{N}{A}{ }^{3} U^{(r)}\right)\left(\partial_{r} \alpha+2 \partial_{r} \beta\right)\right]=0
$$

The total contribution of sources [Eqs. (72) - (75)] that appear in the $3+1$ Einstein equations write in spherical symmetry as follows:

$$
\begin{aligned}
E & =E_{\mathrm{PF}}+E_{\mathrm{R}}, \\
J^{(r)} & =A J^{r}=\left(E_{\mathrm{PF}}+p\right) U^{(r)}+H_{\mathrm{R}}, \\
S_{(r)}^{(r)} & =\left(E_{\mathrm{PF}}+p\right)\left(U^{(r)}\right)^{2}+p+p_{\mathrm{R}}, \\
S_{(\theta)}^{(\theta)} & =S_{(\phi)}^{(\phi)}=p+p_{\mathrm{R}}^{T}, \\
S & =S_{(i)}^{(i)}=\left(E_{\mathrm{PF}}+p\right)\left(U^{(r)}\right)^{2}+3 p+p_{\mathrm{R}}+2 p_{\mathrm{R}}^{T} .
\end{aligned}
$$

\section{GAUGE AND SLICING CONDITION}

Two of the most popular gauges and time slicings that have been used in spherical symmetry are the radial and isotropic gauges and the maximal and polar slicings (see Refs. [49,62.663. for a more general discussion on the slicing choices). Moreover, it is in the framework of asymptotically flat spacetimes (condition usually demanded in astrophysical applications) that they 
become specially useful; it is in this context that such gauges and time slicings will be discussed in the following.

The isotropic gauge $A=B$ with the maximal slicing condition $K=0, \partial_{t} K=0$ (IGMS) has been employed by several authors (e.g. see [6, 10,20,38,40,42]). In the vacuum and static case, that choice leads to the well known Schwarzschild solution in isotropic coordinates. The maximal slicing has the advantage of freezing the evolution in regions near the formation of space-like singularities while allowing a faster evolution in the outer regions (feature usually quoted as "singularity avoidance" property). The time slicing leads to an elliptic equation for the lapse and therefore, for rather general matter conditions (e.g. strong energy condition), one can use the maximum-minimum principle to determine the qualitative behavior for the lapse (cf. 49, 62]). The lapse function has a minimum at $r=0$ and a maximum at $r \rightarrow \infty$ and during the evolution the minimum tends to zero as $t \rightarrow \infty$ (the collapse of the lapse) halting the propertime separation between neighbouring slices as the singularity forms. However, far from the origin $N \rightarrow 1$ which allows to advance the evolution in the asymptotic regions. The IGMS coordinates have also the advantage that the three-metric remains regular at the formation of apparent horizons, which allows to continue the evolution. The drawback is that eventually the metric potencial $A$ grows exponentially at the origin and then the coordinates are "sucked down" to the black hole, which avoids a good description of the evolution outside the event horizon.

The radial gauge $B=1$ with the polar slicing condition (RGPS) $K=K_{r}^{r}$ has been employed sistematically by Gourgoulhon [8,9,36]. These coordinates are a generalization of the Schwarszschild coordinates to the non-static and non-vacuum spacetimes. The field equations turns to be much more simpler than those of the IGMS coordinates since the equations for $A$ and $N$ reduce to first order in $r$ while evolving in time through their sources. Furthermore, the shift $N^{r}$ is zero everywhere on the slices. The RGPS coordinates has a central "singularity avoidance" property which is even stronger that the IGMS coordinates. In fact, the slowing of the evolution is such that it avoids the formation of apparent horizons, the metric 
potential $A$, however, diverges at the star's surface as the matter enters the Schwarzschild radius [8,11], leading, unlike the IGMS to a coordinate crash. Thus, these coordinates do not serve to describe the black hole interior. Nevertheless, the pathological behavior of the RGPS coordinates occurs at a large $t$, and from the astrophysical point of view (e.g., from the point of view of an observer at spatial infinity), these coordinates are good enough to describe the entire evolution of matter outside the black hole, the ingoing matter takes by the way, for an observer at infinity, an infinite time to cross the event horizon (the evolution is thus "frozen").

The hybrid choice of isotropic gauge and polar slicing (IGPS) has been less used in the past. However, it seems that they overcome the drawbacks of the above coordinate choices (cf. 11.40]; see also Ref. [63] for an analysis of these coordinates in the context of axisymmetry).

Another popular gauge choice is the comovil gauge (Lagrangian gauge). This gauge has been particularly used in the study of supernovae collapse with syncronous [13,24, 64 66] and polar slicings [7,61. The comovil coordinates have the advantage that the hydrodynamic equations are simpler since there is no advection. The synchronous slicing are orthogonal in the sense that there is no shift $\left(N^{r}=0\right)$. The disadvantage of the latter is that they fail badly when black holes start forming (cf. [7,67]). The asynchronous coordinates can remedy this problem. In particular Schinder and coauthors [7,61], have used polar slincings to avoid the pathologies of the synchronous gauge. Another modification of the comovil and syncronous coordinates that allows to handle the formation of black holes is the introduction of an outgoing null coordinate instead of the usual time coordinate [27,68].

Finally, we mention the isotropic gauge and constant-mean-curvature slicings (IGKt-slicings) $K=K(t)$, employed by Harleston and coauthoros 44,45]. This time slicing contains the maximal slicig as a particular case. It also posseses the feature of strong crushing coordinate avoidance. These coordinates generalize (to the non-homogenoues case) the comoving coordinates of homogeneous and isotropic spacetimes which are relevant in the standard cosmology. Such a choice is thus useful when the space-time is required to be asymp- 
totically Friedmann-Robertson-Walker [45, 44]. The main difference between this choice and the maximal slicing condition is thus the behavior of the hipersurfaces asymptotically: the maximal hypersurfaces reach spatial infinity while the $K_{t}$-hypersurfaces reach future or past null infinity whether $K$ is positive or negative 67.

\section{A. Isotropic gauge and maximal slicing condition (IGMS)}

The isotropic choice $A=B(\alpha=\beta)$, implies from Eq.(129) that

$$
K_{r}^{r}=K_{(r)}^{(r)}=-\frac{1}{N}\left(\partial_{t} \alpha+\partial_{r} N^{r}+N^{r} \partial_{r} \alpha\right)
$$

Therefore

$$
\partial_{t} \alpha=-N K_{r}^{r}-\partial_{r} N^{r}-N^{r} \partial_{r} \alpha .
$$

On the other hand, the maximal slicing condition $K=0$ implies that

$$
K_{r}^{r}=-2 K_{\theta}^{\theta},
$$

or equivalently

$$
3 \partial_{t} \alpha+3 N^{r} \partial_{r} \alpha+\partial_{r} N^{r}+\frac{2 N^{r}}{r}=0 .
$$

Using (207) in previous Eq. we obtain

$$
\partial_{r} N^{r}-\frac{N^{r}}{r}=-\frac{3}{2} N K_{r}^{r},
$$

This can be written as to give the following differential equation for $N^{r}$ [cf. Eq.(21) of Shapiro \& Teukolsky (1980) [6]],

$$
\partial_{r}\left(\frac{N^{r}}{r}\right)=-\frac{3}{2 r} N K_{r}^{r} .
$$

On the other hand, using Eq. (210) in Eq. (207) one obtains an evolution equation for $\alpha$ :

$$
\partial_{t} \alpha=-N^{r}\left(\partial_{r} \alpha+\frac{1}{r}\right)+\frac{1}{2} N K_{r}^{r} .
$$


With the above choice and with (208), the Hamiltonian constraint Eq. (134) reads,

$$
2 \partial_{r r}^{2} \alpha+\left(\partial_{r} \alpha\right)^{2}+\frac{4 \partial_{r} \alpha}{r}=-8 \pi G_{0} E A^{2}-\frac{3}{4} A^{2}\left(K_{r}^{r}\right)^{2}
$$

Adopting the variable

$$
\tilde{\alpha}=\alpha / 2
$$

we obtain a second order differential equation for $\tilde{\alpha}$

$$
\partial_{r r}^{2} \tilde{\alpha}+\frac{2 \partial_{r} \tilde{\alpha}}{r}=-A^{2}\left[2 \pi G_{0} E+\frac{3}{16}\left(K_{r}^{r}\right)^{2}\right]-\left(\partial_{r} \tilde{\alpha}\right)^{2}
$$

where we recognize in the 1.h.s the Laplacian operator of a spherically symmetric Euclidean space [cf. Eq.(19) of Shapiro \& Teukolsky (1980) [6] for a source term $E$ including a perfect fluid alone Eq.(141)].

The momentum constraint Eq.(136), reads

$$
3 K_{r}^{r}\left(\frac{1}{r}+\partial_{r} \alpha\right)+\partial_{r} K_{r}^{r}=8 \pi G_{0} J_{r}
$$

were we used $J_{r}=A J_{(r)}$. This can be written as a differental equation for $K_{r}^{r}$ as [cf. Eq.(20) of Shapiro \& Teukolsky (1980) [6]],

$$
\partial_{r}\left(A^{3} r^{3} K_{r}^{r}\right)=8 \pi G_{0} r^{3} A^{3} J_{r}
$$

The Eq.(139) provides an elliptic equation for $N$ [cf. Eq. (18) of Shapiro \& Teukolsky (1980) [6] for source terms $E$ and $S$ of a perfect fluid Eqs. (141) and (142)],

$$
\partial_{r r}^{2} \nu+\left(\partial_{r} \nu\right)^{2}+\left(\partial_{r} \alpha\right)\left(\partial_{r} \nu\right)+\frac{2 \partial_{r} \nu}{r}=4 \pi G_{0} A^{2}(S+E)+\frac{3}{2} A^{2}\left(K_{r}^{r}\right)^{2}
$$

We have then four differential Eqs. (211), (215), (216) and (218) for $N^{r}, A$ and $K_{r}^{r}$ y $N$ respectively. It is to note in those equations that the field variables evolve in time through the matter fields. Although the evolution equation for $K_{r}^{r}$ is redundant, for completness we write it in the IGMS coordinates. Equations (137), (213) and (218), lead to

$$
\partial_{t} K_{(r)}^{(r)}+\frac{N^{(r)} \partial_{r} K_{(r)}^{(r)}}{A}+\frac{3}{4} N\left(K_{(r)}^{(r)}\right)^{2}-\frac{N}{A^{2}}\left[\left(\partial_{r} \nu+\partial_{r} \alpha\right)\left(\frac{2}{r}+\partial_{r} \alpha\right)+\left(\partial_{r} \nu\right)\left(\partial_{r} \alpha\right)\right]=-8 \pi G_{0} N S_{(r)}^{(r)}
$$


Concerning the energy conservation equation (148), this reads,

$\partial_{t} E_{\mathrm{PF}}+N^{r} \partial_{r} E_{\mathrm{PF}}=-N \partial_{r} J_{\mathrm{PF}}^{r}-N J_{\mathrm{PF}}^{r}\left(2 \partial_{r} \nu+\frac{2}{r}+3 \partial_{r} \alpha\right)+N K_{(r)}^{(r)}\left(\operatorname{PF}_{(r)}^{(r)}-{ }_{\mathrm{PF}} S_{(\theta)}^{(\theta)}\right)-N^{2} \mathcal{F}^{t}$.

Or in terms of $J_{r}=A^{2} J^{r}$ we obtain

$\partial_{t} E_{\mathrm{PF}}+N^{r} \partial_{r} E_{\mathrm{PF}}=-\frac{N}{A^{2}} \partial_{r} J_{r}^{\mathrm{PF}}-\frac{N}{A^{2}} J_{r}^{\mathrm{PF}}\left(2 \partial_{r} \nu+\frac{2}{r}+\partial_{r} \alpha\right)+N K_{(r)}^{(r)}\left(\mathrm{PF}_{(r)}^{(r)}-{ }_{\mathrm{PF}} S_{(\theta)}^{(\theta)}\right)-N^{2} \mathcal{F}_{t}$.

The conservative form Eq. 150 writes in this gauge as

$$
\begin{aligned}
\partial_{t} E_{\mathrm{PF}}+\frac{1}{r^{2}} \partial_{r}\left(r^{2} V^{r} E_{\mathrm{PF}}\right)= & E_{\mathrm{PF}}\left(\frac{2 N^{r}}{r}+\partial_{r} N^{r}\right)-\frac{1}{r^{2}} \partial_{r}\left(r^{2} \frac{N}{A}{ }^{3} U^{(r)} p\right) \\
& -\frac{N J_{(r)}^{\mathrm{PF}}}{A}\left[\partial_{r} \nu+3 \partial_{r} \alpha-{ }^{3} U^{(r)} A K_{(r)}^{(r)}+N A \mathcal{F}^{t}\right] .
\end{aligned}
$$

The momentum conservation equations writes

$$
\begin{aligned}
& \partial_{t} J_{r}^{\mathrm{PF}}+N^{r} \partial_{r} J_{r}^{\mathrm{PF}}=-J_{r}^{\mathrm{PF}} \partial_{r} N^{r}-N\left[\partial_{r} \mathrm{PF}_{(r)}^{(r)}+2\left(\operatorname{PF}_{(r)}^{(r)}-\operatorname{PF} S_{(\theta)}^{(\theta)}\right)\left(\frac{1}{r}+\partial_{r} \alpha\right)\right. \\
& \left.+\left({ }_{\mathrm{PF}} S_{(r)}^{(r)}+E_{\mathrm{PF}}\right) \partial_{r} \nu+{ }^{3} \mathcal{F}_{r}\right] \text {. }
\end{aligned}
$$

In terms of triad components Eq. (156) reads,

$$
\begin{aligned}
\partial_{t} J_{(r)}^{\mathrm{PF}}+\frac{1}{r^{2}} \partial_{r}\left(r^{2} V^{r} J_{(r)}^{\mathrm{PF}}\right)= & J_{(r)}^{\mathrm{PF}}\left[N K_{(r)}^{(r)}-3 V^{r} \partial_{r} \alpha+N^{r}\left(3 \partial_{r} \alpha-\frac{2}{r}\right)-\partial_{r} N^{r}\right] \\
& -\frac{N}{A}\left[\left(E_{\mathrm{PF}}+p\right) \partial_{r} \nu+\partial_{r} p+{ }^{3} \mathcal{F}_{(r)} A\right] .
\end{aligned}
$$

In this gauge, the Euler equation (157) reads

$$
\begin{gathered}
\partial_{(t)}{ }^{3} U^{(r)}+{ }^{3} U^{(r)}{ }^{3} \partial_{(r)}{ }^{3} U^{(r)}=-\frac{1}{E_{\mathrm{PF}}+p}\left[{ }^{3} \partial^{(r)} p+{ }^{3} U^{(r)} \partial_{(t)} p\right] \\
+\frac{1}{\Gamma^{2}}\left({ }^{3} U^{(r)} K_{(r)}^{(r)}-{ }^{3} \partial_{(r)} \nu\right)+\frac{1}{E_{\mathrm{PF}}+p}\left({ }^{3} U^{(r)} \mathcal{F}^{(t)}-\mathcal{F}^{(r)}\right) .
\end{gathered}
$$

Under the IGMS coordinates the evolution equations for the entropy per baryon and the particle number per baryon keep the same form as Eqs. (195) and (197) or the arternative form given by Eqs. (196) and (198), where as Eqs. (193) and (194) read

$$
\partial_{t}\left(A^{3} n_{E}\right)+\frac{1}{r^{2}} \partial_{r}\left[r^{2} V^{r} n_{E} A^{3}\right]=0
$$




$$
\partial_{t}\left(A^{3} n_{E}\right)+\frac{1}{r^{2}} \partial_{r}\left[A^{3} r^{2} n_{E}\left(N^{r}+\frac{N}{A}{ }^{3} U^{(r)}\right)\right]=0
$$

The equation (226) has a conservative form for the quantity $n_{\mathrm{E}} A^{3}$.

When using the evolution equation (212), the Eqs. (226) and 227) become respectively

$$
\begin{gathered}
\partial_{t} n_{\mathrm{E}}+\frac{1}{r^{2}} \partial_{r}\left[r^{2} V^{r} n_{\mathrm{E}}\right]+3 n_{\mathrm{E}}\left[\left(V^{r}-N^{r}\right) \partial_{r} \alpha-\frac{N^{r}}{r}+\frac{1}{2} N K_{r}^{r}{ }_{r}\right]=0, \\
\partial_{t} n_{\mathrm{E}}+\frac{1}{r^{2}} \partial_{r}\left[r^{2} n_{\mathrm{E}}\left(N^{r}+\frac{N}{A}{ }^{3} U^{(r)}\right)\right]+3 n_{\mathrm{E}}\left[\frac{N}{A}{ }^{3} U^{(r)} \partial_{r} \alpha-\frac{N^{r}}{r}+\frac{1}{2} N K_{r}^{r}\right]=0 .
\end{gathered}
$$

The equation (228) has a conservative form for the quantity $n_{E}$.

\section{B. Radial gauge and polar slicing condition (RGPS)}

In this gauge, $B=1(\beta=0)$ and the polar slicing condition $K=K_{r}^{r}$ is equivalent to $K^{\theta}{ }_{\theta}+K_{\phi}^{\phi}=0$. Since $K_{\theta}^{\theta}=K_{\phi}^{\phi}$, this slicing condition and the gauge choice lead to $N^{r}=0$.

The Hamiltonian constraint Eq.(134) reads

$$
\frac{1}{r^{2}}\left(A^{2}-1\right)+\frac{2}{r} \partial_{r} \alpha=8 \pi G_{0} E A^{2}
$$

Moreover, by defining

$$
A(r, t):=\left(1-\frac{2 G_{0} m(r, t)}{r}\right)^{-1 / 2}
$$

the Eq.(230) reads,

$$
\partial_{r} \alpha=A^{2} G_{0}\left(4 \pi r E-\frac{m}{r^{2}}\right)
$$

or even [cf. Eq.(18) of Ref. [8] or Eq.(3.29) of Ref. [36] for a perfect fluid alone or for a perfect fluid accompanied by a neutrino flow, respectively],

$$
\partial_{r} m=4 \pi r^{2} E
$$

The momentum constraint (135), for the present gauge choice reads 


$$
K_{(r)}^{(r)}=-\frac{1}{A N} \partial_{t} A=4 \pi r G_{0} J_{r}=4 \pi r G_{0} A^{2} J^{r}=4 \pi r G_{0} A J^{(r)}
$$

This with Eq. (231) results in an evolution equation for $m(r, t)$ [cf. Eq.(20) of Ref. [8] or Eq.(3.31) of Ref. [36] for a perfect fluid alone or for a perfect fluid accompanied by a neutrino flow, respectively]:

$$
\partial_{t} m=-4 \pi r^{2} N J^{r}
$$

The evolution equation (138) gives,

$$
\frac{\partial_{r} \nu}{r}-\left[\frac{1}{r^{2}}\left(A^{2}-1\right)+\frac{\partial_{r} A}{r A}\right]=4 \pi G_{0} A^{2}\left(S_{(r)}^{(r)}-E\right)
$$

With (231) and (230) this writes [cf. Eq.(22) of Ref. [8] and Eq.(3.32) of Ref. [36] for a source term $S_{(r)}^{(r)}$ of a perfect fluid alone Eq. (142) or that of a perfect fluid accompanied by a neutrino flow (203), respectively],

$$
\partial_{r} \nu=G_{0} A^{2}\left(\frac{m}{r^{2}}+4 \pi r S_{(r)}^{(r)}\right)
$$

Therefore Eqs. (233) and (237) are the field equations for the two variables $A$ and $N$ respectively. These quantities evolve in time through the matter variables. Therefore, as in the IGMS coordinate choice, the evolution equation for $K_{(r)}^{(r)}$ is also redundant in the RGPS coordinates. For completeness we write it using Eq. (137) [cf. Eq.(21) of Ref. 88 for a perfect fluid alone],

$\partial_{t} K_{(r)}^{(r)}-N\left(K_{(r)}^{(r)}\right)^{2}-\frac{N}{A^{2}}\left[\frac{2 \partial_{r} \alpha}{r}-\partial_{r r}^{2} \nu+\left(\partial_{r} \nu\right)\left(\partial_{r} \alpha-\partial_{r} \nu\right)\right]=4 \pi G_{0} N\left(-S_{(r)}^{(r)}+2 S_{(\theta)}^{(\theta)}-E\right)$

Concerning the evolution equations for the matter, Eq.(148) reads,

$$
\partial_{t} E_{\mathrm{PF}}+\frac{N}{A r^{2}} \partial_{r}\left(A r^{2} J_{\mathrm{PF}}^{r}\right)=N K_{(r)}^{(r)}\left(\mathrm{PF}_{(r)}^{(r)}+E_{\mathrm{PF}}\right)-2 J_{\mathrm{PF}}^{r} \partial_{r} N-N^{2} \mathcal{F}^{t}
$$

which can be written as

$$
\partial_{t} E_{\mathrm{PF}}+\frac{1}{r^{2}} \partial_{r}\left(N r^{2} J_{\mathrm{PF}}^{r}\right)=N K_{(r)}^{(r)}\left({ }_{\mathrm{PF}} S_{(r)}^{(r)}+E_{\mathrm{PF}}\right)-N J_{\mathrm{PF}}^{r}\left(\partial_{r} \nu+\partial_{r} \alpha\right)-N^{2} \mathcal{F}^{t}
$$


We can replace the gradients of the metric potentials by using the Eqs. (230) and (236) which imply,

$$
\partial_{r} \nu+\partial_{r} \alpha=4 \pi r G_{0} A^{2}\left(S_{(r)}^{(r)}+E\right)
$$

Then

$\partial_{t} E_{\mathrm{PF}}+\frac{1}{r^{2}} \partial_{r}\left(N r^{2} J_{\mathrm{PF}}^{r}\right)=N K_{(r)}^{(r)}\left({ }_{\mathrm{PF}} S_{(r)}^{(r)}+E_{\mathrm{PF}}\right)-4 \pi r G_{0} N A^{2} J_{\mathrm{PF}}^{r}\left(S_{(r)}^{(r)}+E\right)-N^{2} \mathcal{F}^{t}$.

Using the momentum constraint (234), we obtain

$$
\partial_{t} E_{\mathrm{PF}}+\frac{1}{r^{2}} \partial_{r}\left(N r^{2} J_{\mathrm{PF}}^{r}\right)=4 \pi r G_{0} N A^{2}\left[J^{r}\left(\mathrm{PF}_{(r)}^{(r)}+E_{\mathrm{PF}}\right)-J_{\mathrm{PF}}^{r}\left(S_{(r)}^{(r)}+E\right)\right]-N^{2} \mathcal{F}^{t} .
$$

Now, since

$$
\begin{aligned}
J^{r} & =J_{\mathrm{PF}}^{r}+J_{\mathrm{R}}^{r}, \\
E & =E_{\mathrm{PF}}+E_{\mathrm{R}}, \\
S_{(r)}^{(r)} & ={ }_{\mathrm{PF}} S_{(r)}^{(r)}+{ }_{\mathrm{R}} S_{(r)}^{(r)},
\end{aligned}
$$

We find

$$
\partial_{t} E_{\mathrm{PF}}+\frac{1}{r^{2}} \partial_{r}\left(N r^{2} J_{\mathrm{PF}}^{r}\right)=4 \pi r G_{0} N A^{2}\left[J_{\mathrm{R}}^{r}\left({ }_{\mathrm{PF}} S_{(r)}^{(r)}+E_{\mathrm{PF}}\right)-J_{\mathrm{PF}}^{r}\left({ }_{\mathrm{R}} S_{(r)}^{(r)}+E_{\mathrm{R}}\right)\right]-N^{2} \mathcal{F}^{t} .
$$

Using that $J_{\mathrm{PF}}^{(r)}=A J_{\mathrm{PF}}^{r}, J_{\mathrm{R}}^{(r)}=A J_{\mathrm{R}}^{r}$ and Eqs. (142) and (144), the energy conservation Eq.(247) finally reads [cf. Eq.(25) of Ref. [8] or Eq.(3.55) of Ref. [36] for a perfect fluid alone or for a perfect fluid accompanied by a neutrino flow, respectively],

$\partial_{t} E_{\mathrm{PF}}+\frac{1}{r^{2}} \partial_{r}\left[r^{2}\left(E_{\mathrm{PF}}+p\right) V^{r}\right]=4 \pi r G_{0} N A\left(E_{\mathrm{PF}}+p\right)\left[J_{\mathrm{R}}^{(r)}\left(\left(U^{(r)}\right)^{2}+1\right)-U^{(r)}\left({ }_{\mathrm{R}} S_{(r)}^{(r)}+E_{\mathrm{R}}\right)\right]-N^{2} \mathcal{F}^{t}$

The alternative expression of Eq. (248) in conservative form reads

$$
\begin{aligned}
\partial_{t} E_{\mathrm{PF}}+\frac{1}{r^{2}} \partial_{r}\left(r^{2} V^{r} E_{\mathrm{PF}}\right)= & -\frac{1}{r^{2}} \partial_{r}\left(r^{2} V^{r} p\right)+4 \pi r G_{0} N A\left(E_{\mathrm{PF}}+p\right)\left[J_{\mathrm{R}}^{(r)}\left(\left(U^{(r)}\right)^{2}+1\right)-U^{(r)}\left({ }_{\mathrm{R}} S_{(r)}^{(r)}+E_{\mathrm{R}}\right)\right] \\
& -N^{2} \mathcal{F}^{t}
\end{aligned}
$$

The momentum conservation Eq.(152) reads 
$\partial_{t} J_{r}^{\mathrm{PF}}+N \partial_{r \mathrm{PF}} S_{(r)}^{(r)}+\frac{2 N}{r} \mathrm{PF}\left(S_{(r)}^{(r)}-{ }_{\mathrm{PF}} S_{(\theta)}^{(\theta)}\right)=-\left(\mathrm{PF}_{(r)} S_{(r)}^{(r)}+E_{\mathrm{PF}}\right) \partial_{r} N+N J_{r}^{\mathrm{PF}} K_{(r)}^{(r)}-{ }^{3} \mathcal{F}_{r} N$.

This can be written as

$$
\partial_{t} J_{r}^{\mathrm{PF}}=N\left[-\frac{1}{r^{2} N} \partial_{r}\left(r^{2} N_{\mathrm{PF}} S_{(r)}^{(r)}\right)+\frac{2}{r}{ }_{\mathrm{PF}} S_{(\theta)}^{(\theta)}-E_{\mathrm{PF}} \partial_{r} \nu+J_{r}^{\mathrm{PF}} K_{(r)}^{(r)}-{ }^{3} \mathcal{F}_{r}\right]
$$

Using (156) the conservative form of this latter reads

$$
\partial_{t} J_{(r)}^{\mathrm{PF}}+\frac{1}{r^{2}} \partial_{r}\left(r^{2} V^{r} J_{(r)}^{\mathrm{PF}}\right)=J_{(r)}^{\mathrm{PF}}\left[2 N K_{(r)}^{(r)}-V^{r} \partial_{r} \alpha\right]-\frac{N}{A}\left[\left(E_{\mathrm{PF}}+p\right) \partial_{r} \nu+\partial_{r} p+{ }^{3} \mathcal{F}_{(r)} A\right]
$$

Furthermore, the use of Eqs. (231), (232), 234) and (237) lead to

$$
\begin{aligned}
\partial_{t} J_{(r)}^{\mathrm{PF}}+\frac{1}{r^{2}} \partial_{r}\left(r^{2} V^{r} J_{(r)}^{\mathrm{PF}}\right)= & G_{0} J_{(r)}^{\mathrm{PF}}\left[8 \pi r A N J^{(r)}-A^{2} V^{r}\left(4 \pi r E-\frac{m}{r^{2}}\right)\right] \\
& -G_{0} N A\left(E_{\mathrm{PF}}+p\right)\left(\frac{m}{r^{2}}+4 \pi r S_{(r)}^{(r)}\right)-\frac{N}{A}\left(\partial_{r} p+{ }^{3} \mathcal{F}_{(r)} A\right)
\end{aligned}
$$

Finally, using Eqs. (244) and (246) and the expressions (142) and (144), we obtain

$$
\begin{aligned}
\partial_{t} J_{(r)}^{\mathrm{PF}}+\frac{1}{r^{2}} \partial_{r}\left(r^{2} V^{r} J_{(r)}^{\mathrm{PF}}\right)= & G_{0} J_{(r)}^{\mathrm{PF}}\left\{4 \pi r A N\left(J_{(r)}^{\mathrm{PF}}+2 J_{\mathrm{R}}^{(r)}\right)-A^{2} V^{r}\left[4 \pi r\left(E_{\mathrm{PF}}+E_{\mathrm{R}}\right)-\frac{m}{r^{2}}\right]\right\} \\
& -G_{0} N A\left(E_{\mathrm{PF}}+p\right)\left[\frac{m}{r^{2}}+4 \pi r\left(p+\mathrm{R} S_{(r)}^{(r)}\right)\right]-\frac{N}{A}\left(\partial_{r} p+{ }^{3} \mathcal{F}_{(r)} A\right) .
\end{aligned}
$$

The Euler equation (157) and Eqs. (234) and (237) lead to

$$
\begin{gathered}
\partial_{(t)}{ }^{3} U^{(r)}+{ }^{3} U^{(r)}{ }^{3} \partial_{(r)}{ }^{3} U^{(r)}=-\frac{1}{E_{\mathrm{PF}}+p}\left[{ }^{3} \partial^{(r)} p+{ }^{3} U^{(r)} \partial_{(t)} p\right] \\
-\frac{G_{0} A}{\Gamma^{2}}\left[\frac{m}{r^{2}}+4 \pi r\left(S_{(r)}^{(r)}-{ }^{3} U^{(r)} J_{(r)}\right)\right]+\frac{1}{E_{\mathrm{PF}}+p}\left({ }^{3} U^{(r)} \mathcal{F}^{(t)}-{ }^{3} \mathcal{F}^{(r)}\right) .
\end{gathered}
$$

Again, using Eqs. (244) and (246) and the expressions (142) and (144), we obtain

$$
\begin{aligned}
\partial_{t}{ }^{3} U^{(r)}+V^{r} \partial_{r}{ }^{3} U^{(r)}= & -\frac{1}{\left(E_{\mathrm{PF}}+p\right)}\left(\frac{N}{A} \partial_{r} p+{ }^{3} U^{(r)} \partial_{t} p\right)-\frac{A N G_{0}}{\Gamma^{2}}\left[\frac{m}{r^{2}}+4 \pi r\left(p+{ }_{\mathrm{R}} S_{(r)}^{(r)}-{ }^{3} U^{(r)} J_{\mathrm{R}}^{(r)}\right)\right] \\
& +\frac{1}{\left(E_{\mathrm{PF}}+p\right)}\left({ }^{3} U^{(r)} \mathcal{F}^{(t)}-{ }^{3} \mathcal{F}^{(r)} N\right)
\end{aligned}
$$

where we also used that in this gauge $\partial_{(t)}=1 / N \partial_{t}, \partial_{(r)}=1 / A \partial_{r}$ and $U^{(r)}=\frac{A}{N} V^{r}$.

This is the Euler equation of the fluid in spherical symmetry which includes the forces of the radiation fields acting on the fluid [cf. Eq.(34) of Ref. [8] or Eq.(3.56) of Ref. [36] for a perfect fluid alone or for a perfect fluid accompanied by a neutrino flow, respectively]. 
A relation that turns to be useful in this gauge is obtained by combining Eqs. 234 and (241)

$$
\frac{1}{A N} \partial_{t}\left(A^{2}\right)+\partial_{r} \nu+\partial_{r} \alpha=4 \pi r G_{0} A^{2}\left[S_{(r)}^{(r)}+E-2 J^{(r)}\right]
$$

For example, in the static case and for perfect fluids, this provides a simple relation between the gradients of the metric potentials and the pressure and energy-density of matter. Moreover, outside the star surface the only contributions to the total matter variabels are those of radiated particles. Under the free streaming approximation [cf. Eq.(187)], this implies that the r.h.s of (257) vanishes. This situation was investigated analitically in the past using a different gauge [69,70] and corresponds to the external solution.

Finally, the integrability condition $\partial_{r t}^{2} m=\partial_{t r}^{2} m$ imposed in Eqs. (233) and (235) result in the relationship

$$
\partial_{t} E+\frac{1}{r^{2}} \partial_{r}\left(r^{2} N J^{r}\right)=0
$$

This equation is in fact compatible with the evolution equation for the total energy density of matter.

Indeed, subtracting Eq. (243) or more specifically Eq. (248) from (258) one obtains an evolution equation for the energy-density of radiation $E_{\mathrm{R}}$ [cf. Eq. (191) ].

The evolution equation (113) and the alternative form (194) in RGPS coordinates read respectively

$$
\begin{gathered}
\partial_{t}\left(A n_{\mathrm{E}}\right)+\frac{1}{r^{2}} \partial_{r}\left[r^{2} V^{r} A n_{\mathrm{E}}\right]=0 \\
\partial_{t}\left(A n_{\mathrm{E}}\right)+\frac{1}{r^{2}} \partial_{r}\left(r^{2} n_{\mathrm{E}} N^{3} U^{(r)}\right)=0 .
\end{gathered}
$$

Note that Eq. (259) has a conservative form for the quantity $A n_{\mathrm{E}}$.

Using Eqs. (234), (232), (244), (144) and (146) in Eq. (259) it turns

$$
\partial_{t} n_{\mathrm{E}}+\frac{1}{r^{2}} \partial_{r}\left[r^{2} n_{\mathrm{E}} V^{r}\right]-G_{0} A^{2} n_{\mathrm{E}}\left[V^{r}\left(\frac{m}{r^{2}}+4 \pi r p\right)+4 \pi r N J_{\mathrm{R}}^{r}\right]=0
$$


which provides an equation in conservative form for $n_{\mathrm{E}}$.

In the same way, using Eqs. (234) and (237) in the alternative Eq. 260) we obtain

$$
\partial_{t} n_{\mathrm{E}}+\frac{N}{A r^{2}} \partial_{r}\left(r^{23} U^{(r)} n_{\mathrm{E}}\right)+n_{\mathrm{E}} G_{0} A N\left[-4 \pi r J^{(r)}+{ }^{3} U^{(r)}\left(\frac{m}{r^{2}}+4 \pi r S_{(r)}^{(r)}\right)\right]=0
$$

The evolution Eqs. (195) and (197) do not change in form under the RGPS coordinates. However, the alternative form given by Eqs. (196) and (198) in RGPS coordinates write respectively

$$
\begin{gathered}
\partial_{t} \sigma+\frac{N^{3} U^{(r)}}{A} \partial_{r} \sigma=-\frac{N}{n \Gamma \Theta}\left(\mu^{\mathrm{R}} \mathcal{R}_{\mathrm{R}}+\mathcal{D}_{p}\right) . \\
\partial_{t} x_{\mathrm{R}}+\frac{N^{3} U^{(r)}}{A} \partial_{r} x_{\mathrm{R}}=\frac{N}{n \Gamma} \mathcal{R}_{\mathrm{R}} .
\end{gathered}
$$

\section{Isotropic gauge and polar slicing condition (IGPS)}

The isotropic choice $A=B$ (i.e., $\alpha=\beta$ ), and the polar slicing condition $K^{\theta}{ }_{\theta}+K_{\phi}^{\phi}=0$, implies due to the spherical symmetry that $K_{\theta}^{\theta}=0$. This latter leads to an evolution equation for $\alpha$ :

$$
\partial_{t} \alpha=-N^{r} \partial_{r} \alpha-\frac{N^{r}}{r}
$$

This and the expression for $K_{r}^{r}$ [cf. Eq.(129)] provide an equation for the shift:

$$
\partial_{r}\left(\frac{N^{r}}{r}\right)=-\frac{N}{r} K_{(r)}^{(r)} .
$$

The Hamiltonian constraint (134) leads to

$$
\partial_{r r}^{2} \tilde{\alpha}+\frac{2 \partial_{r} \tilde{\alpha}}{r}=-2 \pi G_{0} A^{2} E-\left(\partial_{r} \tilde{\alpha}\right)^{2}
$$

where,

$$
\tilde{\alpha}=\alpha / 2
$$

The momentum constraint (135) writes, 


$$
K_{(r)}^{(r)}\left(\frac{1}{r}+\partial_{r} \alpha\right)=4 \pi G_{0} A J_{(r)}=4 \pi G_{0} J_{r}
$$

The equation (138) together with (267) provide an equation for the lapse,

$$
\partial_{r} \nu\left(\frac{1}{r}+2 \partial_{r} \tilde{\alpha}\right)=4 \pi G_{0} A^{2} S_{(r)}^{(r)}-2 \partial_{r} \tilde{\alpha}\left(\frac{1}{r}+\partial_{r} \tilde{\alpha}\right)
$$

In the case of a perfect fluid alone, this corresponds to Eq. (9) of Shapiro \& Teukolsky [40], and to Eq.(6) of Schinder et al. [7] where the authors use a "Lagrangian" gauge which can be easily transformed to the IGPS coordinates.

Finally, the evolution equation for $K_{(r)}^{(r)}$ given by Eq. (137) together with Eq. (267) yield

$$
\begin{aligned}
\partial_{t} K_{(r)}^{(r)}+\frac{N^{(r)} \partial_{r} K_{(r)}^{(r)}}{A}-N\left(K_{(r)}^{(r)}\right)^{2} & -\frac{N}{A^{2}}\left[-\partial_{r r}^{2} \nu+\left(\partial_{r} \nu\right)\left(\partial_{r} \alpha-\partial_{r} \nu\right)+\left(\partial_{r} \alpha\right)\left(\frac{2}{r}+2 \partial_{r} \alpha\right)\right] \\
& =4 \pi G_{0} N\left(-S_{(r)}^{(r)}+2 S_{(\theta)}^{(\theta)}+E\right) .
\end{aligned}
$$

The equation of conservation of energy (150) reads in this gauge as follows,

$$
\begin{aligned}
\partial_{t} E_{\mathrm{PF}}+\frac{1}{r^{2}} \partial_{r}\left(r^{2} V^{r} E_{\mathrm{PF}}\right)= & E_{\mathrm{PF}}\left(N K_{(r)}^{(r)}+\frac{2 N^{r}}{r}+\partial_{r} N^{r}\right)-\frac{1}{r^{2}} \partial_{r}\left(r^{2} \frac{N}{A}{ }^{3} U^{(r)} p\right) \\
& -\frac{N J_{(r)}^{\mathrm{PF}}}{A}\left[\partial_{r} \nu+3 \partial_{r} \alpha-{ }^{3} U^{(r)} A K_{(r)}^{(r)}\right]+N K_{(r)}^{(r)} p-N^{2} \mathcal{F}^{t} .
\end{aligned}
$$

The equation of conservation of momentum Eq. (156) reads

$$
\begin{aligned}
\partial_{t} J_{(r)}^{\mathrm{PF}}+\frac{1}{r^{2}} \partial_{r}\left(r^{2} V^{r} J_{(r)}^{\mathrm{PF}}\right)= & J_{(r)}^{\mathrm{PF}}\left[2 N K_{(r)}^{(r)}-3 V^{r} \partial_{r} \alpha++N^{r}\left(3 \partial_{r} \alpha-\frac{2}{r}\right)-\partial_{r} N^{r}\right] \\
& -\frac{N}{A}\left[\left(E_{\mathrm{PF}}+p\right) \partial_{r} \nu+\partial_{r} p+{ }^{3} \mathcal{F}_{(r)} A\right] .
\end{aligned}
$$

The Euler equation (157) in this gauge take the same form of equation (225), and the Eqs. (196), (198) and (194) [see Eqs. (226) and (227)] keep also the same form as in the IGMS coordinates. Altenatively, one can also use the simpler form of Eqs. (195) and (197). Furthermore, when using the evolution equation (265) in Eqs. (199) and (199) in IGPS coordinates we obtain respectively

$$
\begin{gathered}
\partial_{t} n_{E}+\frac{1}{r^{2}} \partial_{r}\left[r^{2} V^{r} n_{E}\right]+3 n_{E}\left[\left(V^{r}-N^{r}\right) \partial_{r} \alpha-\frac{N^{r}}{r}\right]=0 \\
\partial_{t} n_{E}+\frac{1}{r^{2}} \partial_{r}\left[r^{2} n_{E}\left(N^{r}+\frac{N}{A}{ }^{3} U^{(r)}\right)\right]+3 n_{E}\left[\frac{N}{A}{ }^{3} U^{(r)} \partial_{r} \alpha-\frac{N^{r}}{r}\right]=0 .
\end{gathered}
$$




\section{Boundary conditions and initial data}

A typical feature of spherically symmetric spacetimes is that the gravitational field variables can evolve in time through the matter fields. So the initial conditions for the matter variables and the boundary conditions fix automatically the initial values for the gravitational field by solving the constraint equations and the slicing condition equation. For spacetimes with less symmetries one is always forced to solve the dynamic Einstein equations to evolve the gravitational field. Let us thus discuss first the boundary conditions.

We call exterior solution the solution of field equations outside the perfect-fluid domain (usually a compact support). In the present case, it does not correspond to the Schwarzschild vacuum solution since in general, the radiated matter will extend to spatial infinity. Thus, the exterior solution has to be found also numerically. The exterior sources of the field equations will be provided by the energy-momentum tensor of particles [cf. Eq. (176)]. The matter variables of particles will evolve in time through the distribution function. Moreover, outside the star, the radiated particles can interact only with themselves, however, this interaction is rather week in comparison with the interaction inside the star. In a first approximation one can thus neglect such interactions and consider that the particles will follow geodesics; the distribution function will thus remain constant along them.

Regarding the boundary conditions, these are rather regularity and asymptotic conditions. For instance, the regularity and the asymptotic flatness condition for the shift are respectively (see Ref. [63] for a more detailed analysis about regularity and boundary conditions)

$$
\begin{aligned}
N^{r}(t, 0) & =0, \\
N^{r}(t, r)_{r \rightarrow \infty} & \rightarrow 0 .
\end{aligned}
$$

Similar conditions apply for $K_{r}^{r}$. These boundary conditions are enforced from Eqs. (211) and (266):

$$
N^{r}(t, r)=\lambda_{\mathrm{TS}} r \int_{r}^{\infty} \frac{N\left(t, r^{\prime}\right)}{r^{\prime}} K_{(r)}^{(r)}\left(t, r^{\prime}\right) d r^{\prime}
$$


where $\lambda_{\mathrm{TS}}=1,3 / 2$ for the IGPS and IGMS coordinates respectively.

The condition for the lapse at the star's center is such that the asymptotic flatness condition $N \rightarrow 1$ is verified. Therefore

$$
N(t, 0)=N_{c}(t)
$$

with $N_{c}(t)$ such that

$$
N(t, r)_{r \rightarrow \infty} \rightarrow 1
$$

Since apriori this is difficult to enforce, a better strategy consists in rescaling the lapse as $\tilde{N}=N / N_{c}$ so that $\tilde{N}_{c}(t) \equiv 1$, then the values of the true lapse can be recovered by using $N_{c}(t)=1 / \tilde{N}(t, r)_{r \rightarrow \infty}$ where the asymptotic value $\tilde{N}_{\infty}(t):=\tilde{N}(t, r)_{r \rightarrow \infty}$ is found numerically at every time step. This rescaling allows to integrate the equations spatially in only one cycle. The rescaling will not affect the relevant equations of motion for $N$ or $A$ since only the derivatives of $\nu$ appear there [i.e., the Eqs. (215), (218), (230), (236), (267), (270) for $N$ and $A$ in the different gauges are invariant to such a rescaling]. However, this is not true for the shift equation and for the Boltzmann equation where $N$ appears explicitly. However, this does not pose a problem since a simultaneous rescaling $\tilde{N}^{r}=N^{r} / N_{c}$ leaves all equations invariant as well as the boundary conditions for $\tilde{N}^{r}$.

In the case of the RGPS and IGPS coordinates one can find an integral expression for the lapse satisfying the boundary conditions. For instance, from Eq. (237)

$$
\nu(t, r)=G_{0} \int_{0}^{r} A^{2}\left(\frac{m}{r^{\prime 2}}+4 \pi r^{\prime} S_{(r)}^{(r)}\right) d r^{\prime}+\nu(t, 0) .
$$

The asymptotic flatness condition Eq.(280) leads to

$$
\nu(t, r)_{r \rightarrow \infty} \rightarrow 0
$$

Therefore from Eq. (281) and the asymptotic condition one obtains

$$
\nu(t, 0)=-G_{0} \int_{0}^{\infty} A^{2}\left(\frac{m}{r^{\prime 2}}+4 \pi r^{\prime} S_{(r)}^{(r)}\right) d r^{\prime} .
$$


This corresponds precisely to the renormalized value $-\tilde{\nu}_{\infty}$. So finally,

$$
\nu(t, r)=-G_{0} \int_{r}^{\infty} A^{2}\left(\frac{m}{r^{\prime 2}}+4 \pi r^{\prime} S_{(r)}^{(r)}\right) d r^{\prime}
$$

The value for the lapse at the star surface will be provided by

$$
\nu(t, R(t))=-G_{0} \int_{R(t)}^{\infty} A^{2}\left(\frac{m}{r^{\prime 2}}+4 \pi r^{\prime} S_{(r)}^{(r)}\right) d r^{\prime}
$$

where $R(t)$ corresponds to the RGPS-r coordinate at the star surface at time $t$. We emphasize that, outside the $\operatorname{star} S_{(r)}^{(r)}={ }_{\mathrm{R}} S_{(r)}^{(r)}$, that is, the only contribution to $S_{(r)}^{(r)}$ is from the radiated particles. In fact, outside the star we can write,

$$
\begin{aligned}
\nu(t, r)_{\text {out }} & =-G_{0} \int_{r \geq R(t)}^{\infty} A^{2}\left(\frac{m}{r^{\prime 2}}+4 \pi r^{\prime}{ }_{\mathrm{R}} S_{(r)}^{(r)}\right) d r^{\prime} \\
& =[\ln \mathrm{A}]_{r \geq R(t)}^{\infty}-4 \pi \int_{r \geq R}^{\infty} A^{2} r^{\prime}\left({ }_{\mathrm{R}} S_{(r)}^{(r)}+E_{\mathrm{R}}\right) d r^{\prime} \\
& =\left.\ln A(t, r)^{-1}\right|_{r \geq R(t)}-4 \pi \int_{r \geq R(t)}^{\infty} A^{2} r^{\prime}\left({ }_{\mathrm{R}} S_{(r)}^{(r)}+E_{\mathrm{R}}\right) d r^{\prime} \\
& =\ln \left[1-\frac{2 G_{0} m(t, r)}{r}\right]_{r \geq R(t)}^{1 / 2}-4 \pi \int_{r \geq R(t)}^{\infty} A^{2} r^{\prime}\left({ }_{\mathrm{R}} S_{(r)}^{(r)}+E_{\mathrm{R}}\right) d r^{\prime}
\end{aligned}
$$

where we used Eq.(232) in order to replace the term with $m / r^{\prime 2}$ and also the asymptotic flatness condition on $A$ [cf. Eq.(289) below]. It is to be stressed that in the absence of matter outside the star surface, the first term of Eq.(286) corresponds to the expression for $\nu$ of the Schwarzshild metric (with $\left.m(t, r)\right|_{r \geq R(t)}=M_{*}$ being $M_{*}$ the total mass of the star). However, in the present case there are contributions (due to the energy-density $E_{\mathrm{R}}$ and pressure ${ }_{\mathrm{R}} S_{(r)}^{(r)}$ of particles which fills some part of the space outside the star) which are the responsible for the actual metric to deviate from the Schwarzschild one. These contributions arise from the second term of Eq. (286). In some cases (e.g. the free streaming approximation) numerical analysis show that these deviations are so small so that they can be neglected (cf. [36]).

Deviations of this kind can be appreciated more easily in presence of non trivial scalar fields, for instance in the phenomenon of spontaneous scalarization [71]. Moreover, for $r>R(t)$, the mass function $m(t, r)$ is larger than $m(t, R(t))$ due to the contribution of $E_{\mathrm{R}}$ to the total energy density [cf. Eq. (233) ]. Indeed the mass difference is given by 


$$
\delta m=4 \pi \int_{R(t)}^{r>R(t)} E_{\mathrm{R}} r^{\prime 2} d r^{\prime}
$$

Another way to appreciate such deviations is by noting that Eq. (257) together with the asymptotic conditions imply, in the case of vacuum, the relationship $A N=1$ which is characteristic of the Schwarzschild solution in RGPS coordinates. However, when matter is present outside the star, then $A N \neq 1$ (e.g. see [71), except of course at spatial infinity.

The boundary conditions for $A$ are similar to those for $N$. Therefore

$$
A(t, 0)=A_{c}(t)
$$

with $A_{c}(t)$ such that

$$
A(t, r)_{r \rightarrow \infty} \rightarrow 1
$$

In the case of the RGPS coordinates the reparametrization Eq. 231) imposes the regularity condition

$$
m(t, 0)=0
$$

Since near the origin $m \sim r^{3}$, the reparametrization inforces that $A(t, 0)=1$. Then $\partial_{t} A(t, 0)=$ 0 . The three metric is thus locally flat at the origin. Moreover, provided that the energy-density of sources falls off at least as fast as $1 / r^{4}$ outside the star, the same mass parametrization will drive $A$ to the required asymptotic value. Note, that this behavior of the metric potential $A$ is compatible with the regularity and asymptotic conditions for $K_{r}^{r}$ that we mentioned above. Moreover, these conditions also imply that $J^{r}$ vanish at the origin as well as asymptotically [cf. Eq.(234) ].

In the case of the IGMS and IGPS coordinates one has an elliptic equation for $\tilde{\alpha}$ which is not invariant to a rescaling on $A$. So unlike the RGPS coordinates where $A_{c}=1$, the central value $A_{c}$, should be determined from a shooting method or otherwise in order to satisfy the asymptotic flatness condition. In fact, near the origin $N^{r} \sim r$, therefore from Eq. (212), it turns that at the origin 


$$
\partial_{t} \alpha(t, 0)=\text { const. }
$$

and thus from the definition Eq. (132) and the regularity condition (292) (see below) one concludes that (depending on the sign of the constant) $A(t, 0)$ can grow exponentially [6, 10, 38, 42]. The three metric is thus conformally flat at the origin in the isotropic gauge.

In addition to the previous regularity conditions we have also

$$
\left.\partial_{r} Q\right|_{r=0}=0=\left.Q^{r}\right|_{r=0}
$$

where $Q$ represents collectively the metric potentials and the scalar matter field variables (e.g, $N, A, m, p, \rho, E$, etc.) and $Q^{r}$ tensor field components (e.g $J^{r}, K_{r}^{r}$, etc.).

A convenient way to impose the asymptotic conditions accurately is by compactifying the outer domain with the help of a transformation $u=1 / r$ from the star's surface $r=R$ to spatial infinity. In this way the infinite domain $r \in[R, \infty)$ is mapped to the compact domain $u \in[1 / R, 0)$, so the integration can be performed from $1 / R$ to "zero" with a high degree of accuracy (cf. [71]). Obviously, the field and matter variables for particles are to be matched continously at $R$.

Regarding the distribution function, the regularity condition at the center on the particle's radial energy flux is $H_{\mathrm{R}}=0$ [see Eq. (178)] which means that the average of the particles' radial velocity as measured by the Eulerian observer at the origin is zero (local isotropy at $r=0)$. Same considerations apply for the radial particle-flux-number $j^{(r)}[\mathrm{cf}$. Eq. (190)]. A sufficient condition for $H_{\mathrm{R}}=0=j^{(r)}$ at $r=0$ is

$$
F_{\mathrm{R}}(t, 0, e, \mu)=G_{\mathrm{R}}(t, e, \mu) \text { with } G_{\mathrm{R}}(t, e, \mu)=G_{\mathrm{R}}(t, e,-\mu)
$$

that is, the distribution function being a pair function of $\mu$ at $r=0$, enforces that the integrals given by Eqs. (178) and (190) vanish identically at the origin. This condition ensures in addition that with a suitable form of $G_{\mathrm{R}}(t, e, \mu)$ in the energy domain the energy-density, pressure and density number of particles in the Eulerian frame are finite at the star's center. Usually the assumption of an ideal quantum gas is adopted as initial condition for the particles 
so that the distribution function is isotropic and given by a Fermi-Dirac or Bose-Einstein distribution (whether the particles are Fermions or Bosons) [20, 44, 45. Then the particles energy-density and pressure will be parametrized initially only by the thermodynamic variables like the temperature. In that case $H_{\mathrm{R}}=0$ all over the initial spacelike hypersurface.

The regularity condition for $F_{\mathrm{R}}$ at $r=0$ is like other scalar quantities,

$$
\left.\partial_{r} F_{\mathrm{R}}\right|_{r=0}=0
$$

Another boundary condition is that the inward flux of particles at the star surface is zero. This is imposed by [56],

$$
\left.F_{\mathrm{R}}\right|_{r=R}=0 \text { for }-1 \leq \mu<0
$$

Returning to the initial conditions, the form of these, will characterize first, the type of configuration (e.g., supernova, neutron star, supermassive star, star cluster) and second the dynamics and ultimate fate of the precursor (e.g. protoneutron star, neutron star, black hole). The goal of future numerical investigation will be to explore a large set of initial conditions and their consequences (cf. [72]).

\section{CONCLUSIONS}

Several astrophysical phenomena involve the dynamics of relativistic objects. Some of the most interesting ones end up in the formation of black holes or neutron stars, like the collapse of cores and supernova explosions. While most of the astrophysical objects are rotating, the role of rotation in relativity can be neglected as regards the structure of the object when the rotation frequency is low. Aside from fast pulsars, most of the astrophysical objects are endowed with a low rotation frequency. Therefore, the spherical symmetry is an assumption that can be very useful in a wide range of applications. On the other hand, the mere existence of fast pulsars reveals that rotation has to be taken into acount in a more realistic situation. Moreover, it seems that the deviations from spherical symmetry in supernova explosions is 
central in the phenomenon [17], and that rotation can influence the cooling mechanims in early phases of neutron stars [73]. In general relativity this is a difficult task and only a few attempts have been succeeded within an evolutionary code (see [74 and references therein).

One can separate the problem of the dynamics of relativistic bodies in two sets. The first one involves the formulation, the geometry and the numerics. A convenient coordinate choice and a powerful numerical analysis can allow long term evolutions leading to a better understanding of several physical phenomena. Thus this is a crucial point which has been recognized by almost all the numerical relativists. Investigations of the effect of several gauges and time slicing conditions is always an important issue. One of the aims of this paper was therefore to derive the fundamental equations under different gauges and write them in several forms suitable for different numerical schemes.

The second set, involves the physical approximations used in the model. In the case of gravitational collapse, we have discussed that neutrinos cannot only play an important role in the dynamics but also that the signal carried by them can be fundamental for a better understanding of the underlying physics and as an invaluable imprint of the ultimate fate of the collapsed object. In particular, if neutrinos turn to be massive particles [34], mechanisms like the early black hole formation could be tested [22].

Furthermore, the equation of state at high densities can also lead to different time scale processes during the collapse and the accretion phase. Therefore, it turns necessary to pursue the analysis with the incorporation of the most recent advances in particle and nuclear physics.

While the formalism presented here included only hydrodynamics and transport theory, the equations are quiet general as to include other types of matter like scalar fields, which are very useful in the analysis of critical phenomena.

Our aim for the future investigations is to perform an extensive numerical analysis of several issues discussed here and more generally to analyse the dynamics of spherically symmetric spacetimes with several kind of matter sources. 


\section{ACKNOWLEDGMENTS}

This work has been supported by DGAPA-UNAM, grant No. 112401 and CONACYT, México, grant No. 32551-E. 


\section{REFERENCES}

[1] B. K. Harrison, K.S. Thorne, M. Wakano, and J. A. Wheeler, Gravitation Theory and Gravitational Collapse, The University of Chicago Press, 1965

[2] S. L. Shapiro, and S.A. Teukolsky, Black Holes, White Dwarfs and Neutron Stars (The physics of compact objects), John Wiley \& Sons, New York, 1983

[3] J. R. Oppenheimer, and H. Snyder, Phys. Rev. 56, 455 (1939)

[4] M. Alcubierre, G. Allen, B. Brügmann, E. Seidel, and W.M. Suen, Phys. Rev. D 62, 124011 (2000)

[5] B. Kelly et al., Phys. Rev. D 64, 084013 (2001)

[6] S. L. Shapiro, and S.A. Teukolsky, Astrophys. J. 235, 199 (1980)

[7] P. J. Schinder, S. A. Bludman, and T. Piran, Phys. Rev. D 37, 2722 (1988)

[8] E. Gourgoulhon, Astron. Astrophys. 252, 651 (1991)

[9] E. Gourgoulhon, Ann. Phys. Fr. 18, 1 (1993)

[10] L. I. Petrich, S. L. Shapiro, and S.A. Teukolsky, Phys. Rev. D 31, 2459 (1985)

[11] L. I. Petrich, S. L. Shapiro, and S.A. Teukolsky, Phys. Rev. D 33, 2100 (1986)

[12] S. A. Colgate and R. H. White, Astrophys. J. 143, 626 (1966)

[13] M. M. May and R. H. White, Comput. Phys 7, 219 (1967); ibid Phys. Rev. 141, 1232 (1966)

[14] J. R. Wilson, Astrophys. J. 163, 209 (1971)

[15] R. Mayle, J. R. Wilson, and D. N. Schramm, Astrophys. J. 318, 288 (1987)

[16] R. Saenz, and S. Shapiro, Astrophys. J. 229, 1107 (1979)

[17] A. Burrows, J. Hayes, and B. A. Fryxell, Astrophys. J. 450, 830 (1995)

[18] A. Burrows, Astrophys. J. 334, 891 (1988) 
[19] A. Burrows and J. M. Lattimer, Astrophys. J. 318, L63 (1987)

[20] A. Mezzacappa and R. A. Matzner, Astrophys. J. 343, 853 (1989)

[21] A. Mezzacappa, astro-ph/0010579; ibid astro-ph/0010580

[22] J. F. Beacom, R. N. Boyd, and A. Mezzacappa, Phys. Rev. Lett. 85, 3568 (2000); astro-ph/0010398.

[23] M. Liebendörfer et al., Phys. Rev. D 63, 103004 (2001)

[24] M. Liebendörfer, A. Mezzacappa, and F. K. Thielemann, Phys. Rev. D 63, 104003 (2001)

[25] A. Mezzacappa and S. W. Bruenn, Astrophys. J. 405, 669 (1993); ibid Astrophys. J. 405, 637 (1993), Astrophys. J. 410, 740 (1993)

[26] A. Burrows, Astrophys. J. 334, 891 (1988); ibid Astrophys. J. 300, 488 (1986)

[27] T. W. Baumgarte, S. L. Shapiro, and S.A. Teukolsky, Astrophys. J. 443, 717 (1996)

[28] T. W. Baumgarte, S.A. Teukolsky, S. L. Shapiro, H. T. Janka, and W. Keil, Astrophys. J. 468, 823 (1996)

[29] W. Keil and H. T. Janka, Astron. Astrophys. 271, 187 (1993)

[30] T. W. Baumgarte, S. L. Shapiro, and S.A. Teukolsky, Astrophys. J. 458, 680 (1996)

[31] V. Thorsson, M. Prakash, and J. M. Lattimer, Nucl. Phys. A 572, 693 (1994)

[32] G. E. Brown and H. A. Bethe, Astrophys. J. 423, 659 (1994)

[33] N. K. Glendenning, Astrophys. J. 448, 797 (1995); ibid Astrophys. J. 293, 470 (1985)

[34] Q.R. Ahmad et al., Phys. Rev. Lett. 87, 071301 (2001)

[35] D. B. Cline et al., Phys. Rev. D 50, 720 (1994); R. N. Boyd and A. St. J. Murphy, proceedings of NNN99, AIP Conf Proc 533, (2000) Ed. M Diwan and C.K. Jung, ISBN 1-56396-956-4

[36] E. Gourgoulhon, and P. Haensel, Astron. Astrophys. 271, 187 (1993)

[37] S. L. Shapiro and S.A. Teukolsky, Astrophys. J. 234, L177 (1979) 
[38] S. L. Shapiro and S.A. Teukolsky, Astrophys. J. 298, 34 (1985); ibid Astrophys. J. 298, 58 (1985); ibid Relativistic Stellar Dynamics On the Computer, in Dynamical Spacetimes and Numerical Relativity, (J. M. Centrella ed.), Cambridge University Press, England, 1986.

[39] S. L. Shapiro and S.A. Teukolsky, Astrophys. J. 292, L41 (1985)

[40] S. L. Shapiro and S.A. Teukolsky, Astrophys. J. 307, 575 (1986)

[41] C. S. Kochanek, S. L. Shapiro, and S.A. Teukolsky, Astrophys. J. 320, 73 (1987)

[42] F. A. Rasio, S. L. Shapiro, and S.A. Teukolsky, Astrophys. J. 344, 146 (1989)

[43] F. Linke, J.A. Font, H.T. Janka, E. Müller, and P. Papadopoulos, astro-ph/0103144

[44] H. Harleston, and E.T. Vishniac, Phys. Rev. D 45, 4458 (1992)

[45] H. Harleston, and K. Holcomb, Astrophys. J. 372, 225 (1991)

[46] R. Arnowitt, S. Deser, and C.W. Misner, in Gravitation: an introduction to current research, (L.Witten ed.), Wiley, New York, 1962

[47] Y. Choquet-Bruhat, Cauchy Problem, in Gravitation: an introduction to current research, (L. Witten ed.), Wiley, New York, 1962; Y. Choquet-Bruhat, and J. W. York, The Cauchy Problem, in General Relativity and gravitation: one hundred years after the birth of Albert Einstein, (A. Held ed.), Plenum Press, New York, 1962

[48] M. Salgado, unpublished notes (see http://www.nuclecu.unam.mx/marcelo)

[49] J. W. York, Kinematics and dynamics of general relativity, in Sources of Gravitational Radiation, (L. Smarr ed.), Cambridge University Press, England, 1979.

[50] R. Wald, General Relativity, The University of Chicago Press, Chicago, 1984

[51] S. Chandrasekhar, 1979, in An Einstein Centenary Survey (Hawking S.W, W.Israel eds.), Cambridge University Press, Cambridge, 1979; S. Chandrasekhar, The Mathematical Theory of Black Holes, Oxford University Press, 1983 
[52] M. Salgado, Rev. Mex. Fís. 44, 1 (1998)

[53] M. Salgado, Proc. of the III Workshop of the DGFM-SMF (eds. N. Bretón, O. Pimentel, and J. Socorro), Universidad de Guanajuato, 2000.

[54] S. Bonazzola and E. Gourgoulhon, in Relativistic Gravitation and Gravitational Radiation, Proc. of the Les Houches School of Physics (eds. J. A. Marck and J. P. Lasota), Cambridge University Press, 1997

[55] In order to avoid any misleading when comparing Eq.(48) with Eq. (2.29) of Ref. [54, we point out that the term ${ }^{3} U^{(l)} \mathcal{O}_{(t)(l)}^{(i)}$ of Eq. (48) will arise when transforming the term $n^{\mu} \nabla_{\mu} V^{\alpha}$ of Eq. (2.29) of Ref. 54 to physical components since then $e_{\alpha}^{(\sigma)} n^{\mu} \nabla_{\mu} V^{\alpha}=n^{\mu} \nabla_{\mu} V^{(\sigma)}+n^{(\lambda)} V^{(\beta)} \mathcal{O}_{(\lambda)(\beta)}^{(\sigma)}$. Since $n^{(t)}=1$, and $V^{(t)}=0=n^{(i)}$, then for $\sigma=i$, we have, $n^{(\lambda)} V^{(\beta)} \mathcal{O}_{(\lambda)(\beta)}^{(i)}=V^{(l)} \mathcal{O}_{(t)(l)}^{(i)}$.

[56] R. W. Lindquist, Ann. Phys. 37, 487 (1966)

[57] P. Haensel, Astron. Astrophys. 262, 131 (1992)

[58] R. Duncan, S. Shapiro, and I. Wasserman, Astrophys. J. 267, 358 (1983); ibid, Astrophys. J. 278, 806 (1984)

[59] E. Salpeter and S. Shapiro, Astrophys. J. 251, 311 (1981); P. J. Schinder and S. Shapiro, Astrophys. J. 259, 311 (1982); ibid, Astrophys. J. Supp. 50, 23 (1982); R. Duncan, S. Shapiro, and I. Wasserman, Astrophys. J. 309, 141 (1986)

[60] Most of the algebraic computations which include the $3+1$ form of Einstein equations, matter equations and the Relativistic Boltzmann equation in spherical symmetry were performed by using a MATHEMATICA code presented in M. Salgado, Comp. Phys. Com. 79, 309 (1994).

[61] P. J. Schinder, Phys. Rev. D 38, 1673 (1988)

[62] L. Smarr, and J. W. York, Phys. Rev. D 17, 2529 (1978)

[63] J. M. Bardeen, T. Piran, Phys. Rep. 96, 205 (1983)

[64] C. Misner and D. Sharp, Phys. Rev. 136, 571 (1964) 
[65] E. Baron, E. S. Myra, J. Cooperstein, and L. J. Van Den Horn, Astrophys. J. 339, 978 (1989)

[66] J. Cernohorsky and C. G. Van Weert, Astrophys. J. 398, 190 (1992)

[67] T. Piran, Methods for Numerical Relativity, in Rayonnement Gravitationnel (ed. N. Deruelle and T. Piran), North Holland, 1983

[68] W. C. Hernandez and C. W. Misner, Astrophys. J. 143, 452 (1966)

[69] P. C. Vaidya, Nature 171, 260 (1953)

[70] R.W. Lindquist, R.A. Schwartz, C.W. Misner, Phys. Rev. D 137, B1364 (1965)

[71] M. Salgado, D. Sudarsky, and U. Nucamendi, Phys. Rev. D 58, 124003-1 (1998)

[72] J. Novak, to be published in Astron. Astrophys. (pre-print gr-qc/0107045)

[73] J. A. Miralles, K. A. Van Ripper, and J. M. Lattimer, Astrophys. J. 407, 687 (1993)

[74] M. Shibata, T. W. Baumgarte, and S. L. Shapiro, Phys. Rev. D 61, 044012-1 (2000) 